\title{
Muon spin rotation and neutron scattering investigations of the B-site ordered double perovskite $\mathrm{Sr}_{2} \mathrm{DyRuO}_{6}$
}

D. T. Adroja, Shivani Sharma, C. Ritter, A. D. Hillier, Duc Le, C. V. Tomy, R. Singh, R. I. Smith, M. Koza, A. Sundaresan and S. Langridge

\section{Published version information}

Citation: DT Adroja et al. 'Muon spin rotation and neutron scattering investigations of the B -site ordered double perovskite $\mathrm{Sr}_{2}$ DyRuO $_{6}$.' Physical Review B, vol. 101, no. 9 (2020): 094413.

DOI: $10.1103 /$ PhysRevB.101.094413

This version is made available in accordance with publisher policies. Please cite only the published version using the reference above. This is the citation assigned by the publisher at the time of issuing the APV. Please check the publisher's website for any updates. 


\title{
Muon spin rotation and neutron scattering investigations of the $B$-site ordered double perovskite $\mathrm{Sr}_{2} \mathrm{DyRuO}_{6}$
}

\author{
D. T. Adroja $\odot,{ }^{1,2, *}$ Shivani Sharma,${ }^{1,3, \dagger}$ C. Ritter, ${ }^{4, \ddagger}$ A. D. Hillier, ${ }^{1}$ Duc Le, ${ }^{1}$ C. V. Tomy, ${ }^{5}$ R. Singh $\odot,{ }^{6}$ \\ R. I. Smith $\odot,{ }^{1}$ M. Koza, ${ }^{4}$ A. Sundaresan, ${ }^{3}$ and S. Langridge $\odot^{1}$ \\ ${ }^{1}$ ISIS facility, Rutherford Appleton Laboratory, Chilton, Oxon OX11 OQX, United Kingdom \\ ${ }^{2}$ Highly Correlated Matter Research Group, Physics Department, University of Johannesburg, Auckland Park 2006, South Africa \\ ${ }^{3}$ Jawaharlal Nehru Centre for Advanced Scientific Research, Jakkur, Bangalore 560064, India \\ ${ }^{4}$ Institut Laue-Langevin, 71 Avenue des Martyrs, CS 20156, 38042 Grenoble Cedex 9, France \\ ${ }^{5}$ Department of Physics, Indian Institute of Technology Bombay, Mumbai 400 076, India \\ ${ }^{6}$ Indian Institute of Science Education and Research Bhopal, Bhopal 462066, India
}

(Received 29 July 2019; revised manuscript received 14 December 2019; accepted 11 February 2020; published 11 March 2020)

\begin{abstract}
The magnetic ground state of the $B$-site ordered double perovskite $\mathrm{Sr}_{2} \mathrm{DyRuO}_{6}$ has been investigated using muon spin rotation and relaxation $(\mu \mathrm{SR})$, neutron powder diffraction (NPD), and inelastic neutron scattering (INS), in addition to heat capacity and magnetic susceptibility (AC and DC) measurements. A clear signature of a long-range ordered magnetic ground state has been observed in the heat capacity data, which exhibits two sharp anomalies at 39.5 and $36 \mathrm{~K}$ found as well in the magnetic data. Further supporting evidence consistent with long-range magnetic ordering comes from a sharp drop in the muon initial asymmetry and a peak in the relaxation rate at $40 \mathrm{~K}$, along with a weak anomaly near $36 \mathrm{~K}$. Based on temperature dependent NPD, the low temperature magnetic structure contains two interpenetrating lattices of $\mathrm{Dy}^{3+}$ and $\mathrm{Ru}^{5+}$, forming an antiferromagnetic ground state below $39.5 \mathrm{~K}$ with magnetic propagation vector $k=(0,0,0)$. The magnetic moments of $\mathrm{Dy}^{3+}$ and $\mathrm{Ru}^{5+}$ at $3.5 \mathrm{~K}$ are pointing along the crystallographic $b$ axis with values of $\mu^{\text {Dy }}=4.92(10) \mu_{\mathrm{B}}$ and $\mu^{\mathrm{Ru}}=1.94(7) \mu_{\mathrm{B}}$, respectively. The temperature dependence of the $\mathrm{Ru}^{5+}$ moments follows a mean field type behavior, while that of the $\mathrm{Dy}^{3+}$ moments exhibits a deviation indicating that the primary magnetic ordering is induced by the order of the $4 d$ electrons of $\mathrm{Ru}^{5+}$ rather than that of the proper $4 f \mathrm{Dy}^{3+}$ electrons. The origin of the second anomaly observed in the heat capacity data at $36 \mathrm{~K}$ must be connected to a very small spin reorientation as the NPD studies do not reveal any clear change in the observed magnetic Bragg peaks' positions or intensities between these two transitions. INS measurements reveal the presence of crystal field excitations (CEF) in the paramagnetic state with overall CEF splitting of $73.8 \mathrm{meV}$, in agreement with the point change model calculations, and spin wave excitations below $9 \mathrm{meV}$ at $7 \mathrm{~K}$. Above $T_{N}$, the spin wave excitations transform into a broad diffuse scattering indicating the presence of short-range dynamic magnetic correlations.
\end{abstract}

DOI: 10.1103/PhysRevB.101.094413

\section{INTRODUCTION}

Geometrically frustrated antiferromagnetic (AFM) materials have attracted considerable interest over the past few years, motivated by their tendency to form rather exotic magnetic ground states such as the spin glass, spin liquid, or spin ice instead of long-range magnetic order in apparent defiance of the third law of thermodynamics [1-6]. Among the four "canonical" geometrically frustrated lattices: triangular planar, kagome, pyrochlore, and face-centered cubic (fcc), the latter has recently gained strong attention [6-9]. In real materials, the fcc magnetic lattice is conveniently realized in the $B$-site ordered double perovskites, $A_{2} B B^{\prime} \mathrm{O}_{6}$ [10]. Here a magnetic ion resides on the $B^{\prime}$ site, while $B$ can be either

\footnotetext{
*devashibhai.adroja@stfc.ac.uk

†shivani.sharma@stfc.ac.uk

†ritter@ill.fr
}

magnetic or nonmagnetic and $A$ site is nonmagnetic. Both the $B$ and $B^{\prime}$ sites constitute interpenetrating face-centered cubic sublattices in which, if the exchange constraints between nearest neighbors are AFM, the basic criteria for geometric frustration are satisfied [6,9,11-15].

Recently, the double perovskites compounds with general formula $A_{2} B B^{\prime} \mathrm{O}_{6}$, with $A$ alkaline metals, $B$ rare earth metals, and $B^{\prime}$ transition metal, have attracted considerable attention due to their interesting physical properties as well as possible applications in renewable energy and spintronic devices [6,10-17]. Within this class of materials, there are compounds with properties such as a high Curie temperature $T_{\mathrm{C}}[18,19]$, phase separation [20], a high magnetoresistance $[21,22]$, a metal- insulator transition [23,24], and half-metallic antiferromagnets [16,21]. Besides the interesting fundamental physics, double perovskite materials are important for optoelectronic applications and technology [25]. This huge variety of properties has its origin in the possibility of doping and substituting the perovskite structure at the $A$ and $B$ sites, 
allowing tailoring of the electronic, crystal, and magnetic structure of the compounds, which, in turn, interact with each other. $\mathrm{Sr}_{2} \mathrm{FeMoO}_{6}$ was the first double perovskite for which a high magnetoresistance at room temperature was reported $\left(T_{\mathrm{C}} \sim 420 \mathrm{~K}\right)$ [26]. By electron doping in similar compounds, the Curie temperatures rises to $635 \mathrm{~K}$ for $\mathrm{Sr}_{2} \mathrm{CrReO}_{6}$ [27-29] and $750 \mathrm{~K}$ for $\mathrm{Sr}_{2} \mathrm{CrOsO}_{6}$ [30] which is so far the highest $T_{\mathrm{C}}$ observed in ferrimagnetic double perovskites. A special type of double exchange interactions [31,32] was shown to be responsible for the high magnetic transition temperatures and the strong spin polarization in double perovskites where $B$ and $B^{\prime}$ cations are in a mixed valence state [33]. Adoption of integer valences leads to reduced $T_{\mathrm{C}}$ or to antiferromagnetic order $[33,34]$.

Among the antiferromagnetically ordered double perovskites, $\mathrm{Sr}_{2} \mathrm{LnRuO}_{6}(\mathrm{Ln}=$ rare earth, $\mathrm{Y}, \mathrm{Ho}, \mathrm{Yb}, \mathrm{Lu}$, etc. $)$ compounds exhibit many interesting properties, for example the presence of two magnetic phase transitions and strong geometrical frustration above the magnetic ordering up to as high as $300 \mathrm{~K}$, confirmed via heat capacity and inelastic neutron scattering measurements, respectively [35-39]. Recent neutron diffraction studies on $\mathrm{Sr}_{2} \mathrm{YRuO}_{6}$ reveal that at the first transition temperature only half of the $\mathrm{Ru}$ layers order magnetically while the other half (alternatively) reveals short-range ordering and below the second phase transition the system exhibits a type-I AFM ground state [36]. Although the presence of frustration has been observed in many double perovskite compounds, its origin is not clear at present. In addition, diffuse scattering has been observed in the compounds $\mathrm{Sr}_{2} \mathrm{YRuO}_{6}, \mathrm{La}_{2} \mathrm{NaRuO}_{6}, \mathrm{La}_{2} \mathrm{NaOsO}_{6}$, and $\mathrm{Sr}_{2} \mathrm{YbRuO}_{6}[36,37,40]$ in which $\mathrm{La}_{2} \mathrm{NaRuO}_{6}$ reveals a single magnetic transition below $15 \mathrm{~K}$ to an incommensurate magnetic ground state, whereas $\mathrm{La}_{2} \mathrm{NaOsO}_{6}$ does not exhibit any long-range order down to $4 \mathrm{~K}$ on the quasi-fcc lattices as a result of geometrical frustration [11,40]. These results motivate the investigation of other double perovskite compounds in order to understand the presence of geometrical frustration and its effect on the magnetic ground state. We have therefore studied the detailed dynamic and static magnetic properties of $\mathrm{Sr}_{2} \mathrm{DyRuO}_{6}$ (SDRO) using magnetization, heat capacity, muon spin resonance/rotation $(\mu \mathrm{SR})$, neutron powder diffraction (NPD), and inelastic neutron scattering (INS) measurements. SDRO exhibits a magnetic anomaly at $\sim 40 \mathrm{~K}$, which is suspected to be associated with the longrange ordering $[41,42]$. An exchange bias effect in SDRO has also been observed below the AFM ordering temperature and the possible cause for the observed effect was suspected to be linked to Dzyaloshinskii-Moriya (DM) interactions present in this geometrically frustrated system [41]. DFT results report that the main contribution to the spin moment comes from the $f$ orbitals, with a considerable role of the $d$ orbitals, and suggest that SDRO will behave as a conductor and semiconductor for spin-up and spin-down orientations, respectively [43]. However, no direct evidence or studies about the electronic or magnetic structure/ground state is available on SDRO in the existing literature. The present work will fill the gap to understand the low temperature magnetic behavior of SDRO and provide an ideal example to compare with the available data of other geometrically frustrated double perovskites having two magnetic cations at the $B$ and $B^{\prime}$ sites.

\section{EXPERIMENTAL DETAILS}

The polycrystalline sample ( $10 \mathrm{~g})$ of $\mathrm{Sr}_{2} \mathrm{DyRuO}_{6}$ (SDRO) was prepared by solid-state reaction from stoichiometric amounts of $\mathrm{SrCO}_{3}, \mathrm{RuO}_{2}$, and $\mathrm{Dy}_{2} \mathrm{O}_{3}$ (Aldrich 99.99\%) which were mixed in an agate mortar and pestle, and pressed into pellets. These pellets were then annealed at $1123 \mathrm{~K}$ for 12 $\mathrm{h}$ and sintered at $1253 \mathrm{~K}$ for $24 \mathrm{~h}$, with frequent regrinding and repelletizing. The structure characterization at $300 \mathrm{~K}$ was carried out using the GEM time-of-flight (TOF) neutron powder diffractometer (NPDM) at the ISIS neutron Facility, UK. The DC-magnetic susceptibility and magnetization isotherm were measured using a SQUID magnetometer (Quantum Design, MPMS). Heat capacity measurements were performed using a relaxation technique by a commercial system (Quantum Design, PPMS) in the temperature range of $1.8-100 \mathrm{~K}$. The AC susceptibility was measured using the same Quantum Design PPMS. To investigate the magnetic structure/ground state, low temperature NPD measurements were performed using the constant wavelength $(\lambda=2.396 \AA)$ high intensity diffractometer D20 between 1.7 and $50 \mathrm{~K}$ at ILL Grenoble, France. High-resolution data were recorded as well at the ILL on the powder diffractometer D2B using $\lambda=1.594$ and $2.399 \AA$. All the diffraction data have been analyzed using the Rietveld refinement program Fullprof [44]. The $\mu$ SR experiments were carried out using the MuSR spectrometer in the longitudinal geometry at the ISIS muon facility, UK. We have performed zero-field (ZF) and longitudinal-field (LF) $\mu$ SR measurements between 1.5 and $50 \mathrm{~K}$ and LF field between 0 and $2500 \mathrm{G}$. The powder sample (thickness $\sim 2 \mathrm{~mm}$ ) was mounted onto a $99.995+\%$ pure silver plate using GE varnish and was covered with $18 \mu \mathrm{m}$ silver foil. Inelastic neutron scattering measurements were performed on the time-of-flight spectrometers MERLIN at ISIS Facility and IN6 at ILL, Grenoble. We used a powder sample of SDRO in an annular Al can of outer diameter $40 \mathrm{~mm}$ on MERLIN and $20 \mathrm{~mm}$ on IN6.

\section{RESULTS AND DISCUSSION}

\section{A. Room temperature structural characterization}

Figure 1 shows the NPD pattern of SDRO collected at $300 \mathrm{~K}$ from the $34.96^{\circ}$ detector bank of the GEM diffractometer. The structure was refined using the monoclinic space group $P 2_{1} / n$, assuming a $1: 1$ ordering of the $\mathrm{Dy}^{3+}$ and $\mathrm{Ru}^{5+}$ cations. The $\mathrm{Dy}^{3+}$ and $\mathrm{Ru}^{5+}$ cations occupy distinct Wyckoff sites $2 c$ and $2 d$, respectively, resulting in the ordered arrangement. No impurity peaks were detected within the resolution limit. The refined lattice parameters at 300 $\mathrm{K}$ are $a=5.7774(2) \AA, b=5.7948(2) \AA, c=8.1848(2) \AA$, $\beta=90.181(3)^{\circ}$, and $V=276.88(1) \AA^{3}$. The determined lattice and structural parameters are in good agreement with the existing literature [41-43]. The refinement did not give any evidence for a possible site disorder between the Dy ${ }^{3+}$ and $\mathrm{Ru}^{5+}$ cations. Therefore, our results confirm the ordered double perovskite structure of SDRO.

\section{B. Physical properties}

Figure 2 shows the measured heat capacity of SDRO as a function of temperature for zero field (ZF) and in applied 


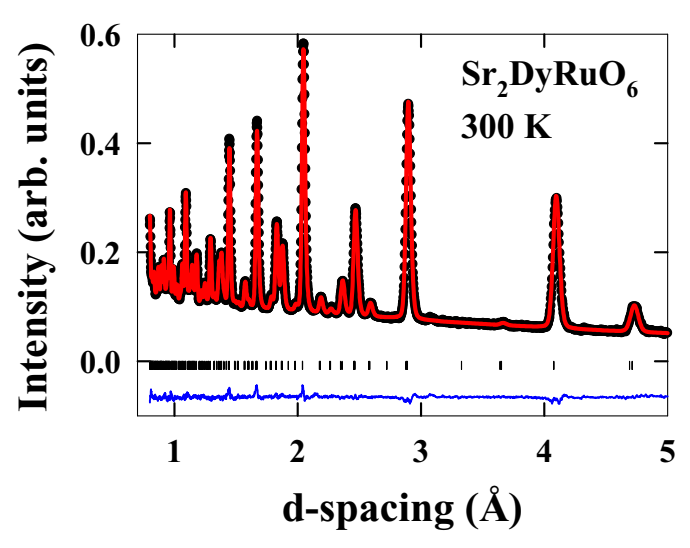

FIG. 1. Rietveld fit to NPD patterns collected at $300 \mathrm{~K}$ on GEM diffractometer at ISIS: Black crosses show observed data points; the red line shows the calculated profile; the lower blue line is the difference profile (obs. - calc.); black vertical markers indicate Bragg peak positions.

fields of 1 and $9 \mathrm{~T}$. Two anomalies are evident at 39.5 and $36 \mathrm{~K}$ (see the inset in Fig. 2) in the ZF heat capacity data, which disappear in a field of $9 \mathrm{~T}$. The anomalies are more clearly visible in the first order derivative which is presented as an inset in the same figure. At $1 \mathrm{~T}$ field, the lower transition does not change much, but the higher transition broadens and moves towards higher temperature. Similar two anomalies/transitions were also reported for isostructural $\mathrm{Sr}_{2} \mathrm{LnRuO}_{6}(\mathrm{Ln}=\mathrm{Y}$, $\mathrm{Ho}, \mathrm{Yb}$, and $\mathrm{Tb}$ ) and identified as antiferromagnetic ordering temperatures $\left(T_{N 2}\right.$ and $\left.T_{N 1}\right)$. The anomalies were situated at 24 and $29 \mathrm{~K}$ for $\mathrm{Y}$ [35,36], 36 and $40 \mathrm{~K}$ for $\mathrm{Yb}$ [37], 32 and $26 \mathrm{~K}$ for $\mathrm{Lu}$ [45], and 15 and $36 \mathrm{~K}$ for Ho-based systems $[38,46]$. On the other hand, the heat capacity study on the cubic $\mathrm{Ba}_{2} \mathrm{DyRuO}_{6}$ reveals only a single anomaly at $47 \mathrm{~K}$ [47], similar to $\mathrm{La}_{2} \mathrm{NaRuO}_{6}[11,40]$. Furthermore, $\mathrm{Sr}_{2} \mathrm{FeOsO}_{6}$ exhibits two magnetic transitions at $T_{N 1}=140 \mathrm{~K}$ and $T_{N 2}=$ $67 \mathrm{~K}$, where both the Fe and Os moments order and the second transition is associated with the change in magnetic structure from $\mathrm{AF} 1$ to $\mathrm{AF} 2$ [48]. Considering the observation of a spin

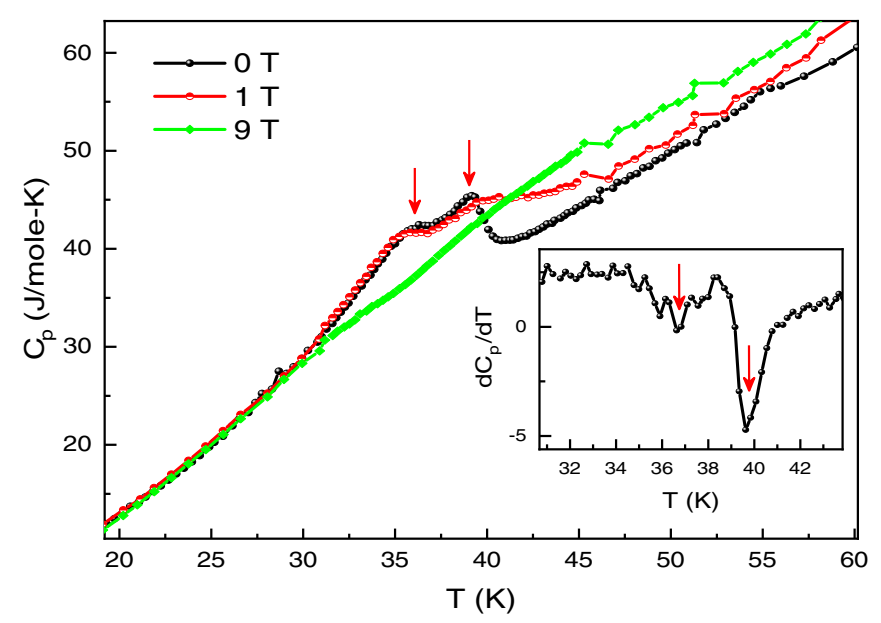

FIG. 2. Heat capacity measurements in the low temperature range measured in zero field and applied field of 1 and $9 \mathrm{~T}$. The inset shows $d C_{\mathrm{p}} / d T$ vs $\mathrm{T}$ near the magnetic ordering.

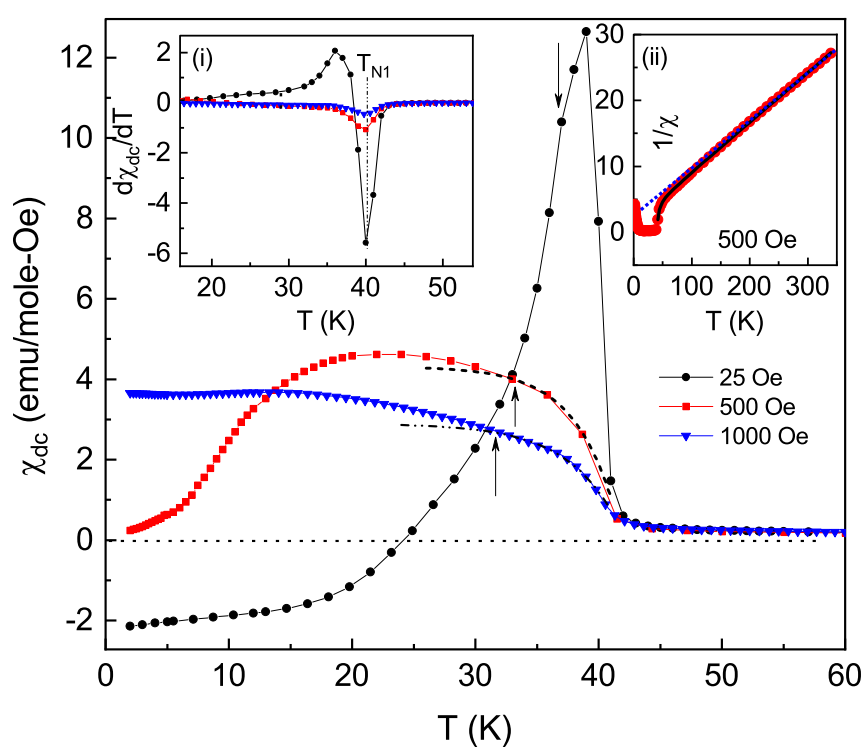

FIG. 3. (a) DC magnetic susceptibility $\left(\chi_{\mathrm{DC}}\right)$ measured at various applied magnetic fields. The dashed and dotted lines are guides to the eye. Inset (i) shows the first derivative of ZFC $\chi_{\mathrm{DC}}$ and the inset (ii) shows the inverse susceptibility $\left(1 / \chi_{\mathrm{DC}}\right)$ measured in an applied field of 500 Oe. The dotted blue line shows the fit to Curie-Weiss behavior and the solid black line shows the fit using the two-level paramagnetic model (see text). It is to be noted that the negative magnetization seen below $25 \mathrm{~K}$ in the 25 Oe data is an artifact due to trapped field in the superconducting magnet of the SQUID magnetometer [74].

gap only below $T_{N 2}$ in the inelastic study of $\mathrm{Sr}_{2} \mathrm{FeOsO}_{6}$, it was suggested that spin-orbit coupling is important for ground state selection in this compound [48]. This suggests that the two anomalies observed in the heat capacity of SDRO are possibly associated either to the separate long-range magnetic ordering of the $\mathrm{Ru}$ and $\mathrm{Dy}$ moments or to a spin reorientation transition.

The temperature dependence of the DC-magnetic susceptibilities $\left(\chi_{\mathrm{DC}}\right)$ of SDRO in various applied magnetic fields is shown in Fig. 3 in zero-field cooled (ZFC). The increase of susceptibility below $42 \mathrm{~K}$, irrespective of the applied field value, indicates the emergence of long-range magnetic ordering. With further decreasing temperature, $\chi_{\mathrm{DC}}$ first increases and exhibits a sharp peak near $40 \mathrm{~K}$ for $B=25 \mathrm{Oe}$, nearly matching the first anomaly observed in the heat capacity data $(39.5 \mathrm{~K})$. For $B=500 \mathrm{Oe}$, the peak in the susceptibility becomes quite broad exhibiting a plateau. For $B=1000$ Oe, there is no visible peak and the susceptibility keeps on increasing down to $2 \mathrm{~K}$. As it is difficult to identify the magnetic ordering temperature directly from the $\chi_{\mathrm{DC}}$ behavior, the first derivative of $\chi_{\mathrm{DC}}$ is plotted in the left inset of the same figure and shows for all three field values a clear peak at $40 \mathrm{~K}$. This is in accordance with the heat capacity results where the first anomaly was observed at $T_{N 1}=39.5 \mathrm{~K}$. No direct signature of a second anomaly as found in the heat capacity data $\left(T_{N 2}=36.5 \mathrm{~K}\right)$ is evident from the susceptibility data (Fig. 3) for $B=500$ and 1000 Oe while the derivative points to $T_{N 2}=36 \mathrm{~K}$ for $B=25 \mathrm{Oe}$ (inset of Fig. 3). Only an indirect indication of $T_{N 2}$ can be found by the rate of change of $\chi_{\mathrm{DC}}$ for 


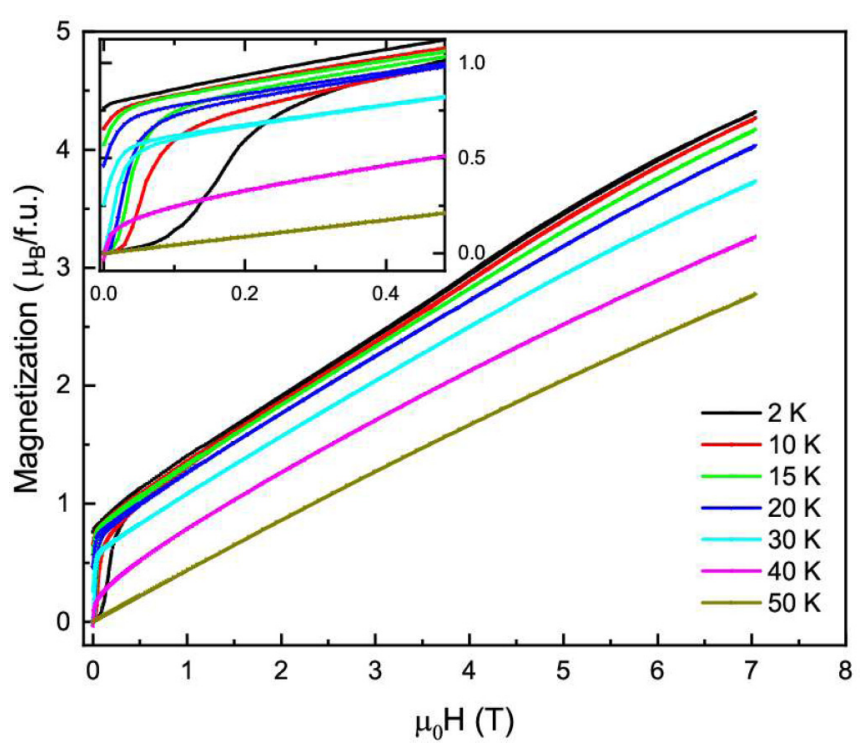

FIG. 4. Magnetization isotherms measured at various temperatures ranging from 2 to $50 \mathrm{~K}$. The inset shows the enlarged view at lower fields. A clear hysteresis can be seen for $T<40 \mathrm{~K}$.

the $B=500$ and 1000 Oe field curves which changes below 35 K. In Fig. 3 the changes in slopes of the magnetic susceptibility are presented by the dashed black lines and using black arrows. As a function of the applied field strength, the values of $T_{N 2}$ can be estimated as $36.7,34$, and $32 \mathrm{~K}$ for $B=25,500$, and 1000 Oe, respectively, The Curie-Weiss (CW) fit of the inverse susceptibility for $500 \mathrm{Oe}$ data is also shown in the same figure in the right inset (dotted blue line), which gives $C=$ $13.56(\mathrm{~K} \mathrm{emu} / \mathrm{mol} \mathrm{Oe})$ and a $\mathrm{CW}$-paramagnetic temperature of $\theta_{\mathrm{CW}}=-27.5 \mathrm{~K}$. The estimated total value of the effective paramagnetic moment is $10.42 \mu_{\mathrm{B}}$ which is slightly smaller than the theoretical value arising from the combined paramagnetic contribution of $\mathrm{Dy}^{3+}$ and $\mathrm{Ru}^{5+}$ ions which amounts to $11.33 \mu_{\mathrm{B}}\left(\mu_{\mathrm{eff}}=\sqrt{\left(\mu_{\mathrm{eff}}^{\text {Dy3 }+}\right)^{2}+\left(\mu_{\mathrm{eff}}^{\mathrm{Ru}+}\right)^{2}}\right)$. Furthermore, we have also fitted the inverse susceptibility of SDRO using a two-sublattice paramagnetic model $[49,50], \chi^{-1}(T)=$ $T / C_{m}+1 / \chi_{0}-b /\left(T-\theta_{p}\right)$, which gives an excellent agreement with the data above $T_{N 1}$ (see solid line in inset (ii) of Fig. 3), with fitting parameters $C_{m}=13.30 \mathrm{~K} \mathrm{emu} / \mathrm{mol}, \chi_{0}=$ $0.58(\mathrm{emu} / \mathrm{mol}), b=10.59 \mathrm{~K} \mathrm{~mol} / \mathrm{emu}$, and $\theta_{p}=37.88 \mathrm{~K}$.

The isothermal magnetization behavior ( $M$ vs $H$ ) of SDRO is presented in Fig. 4 as a function of applied magnetic field $(H)$ at selected temperatures ranging from 2 to $50 \mathrm{~K}$. To perform these measurements, the sample was cooled each time from the paramagnetic state $(300 \mathrm{~K})$ to avoid any magnetic history effect. The $M$ vs $H$ isotherm at $50 \mathrm{~K}$ is almost linear in $H$, as expected for a paramagnet state. At $2 \mathrm{~K}$, the $M$ vs $H$ curve initially increases rapidly with increasing field up to $0.4 \mathrm{~T}$ before it exhibits an almost linear field dependence. The rapid increase in magnetization is also observed for temperatures between 2 and $40 \mathrm{~K}$, but the value above which the field dependence is showing a linear behavior is reducing with increasing temperature. The observed weak ferromagnetictype behavior in $M$ vs $H$ data at low fields in the antiferromagnetic state, which is also observed in other $\mathrm{Sr}_{2} \mathrm{LnRuO}_{6}$ ( $\mathrm{R}=$ rare earth) compounds [37-39], has been attributed to

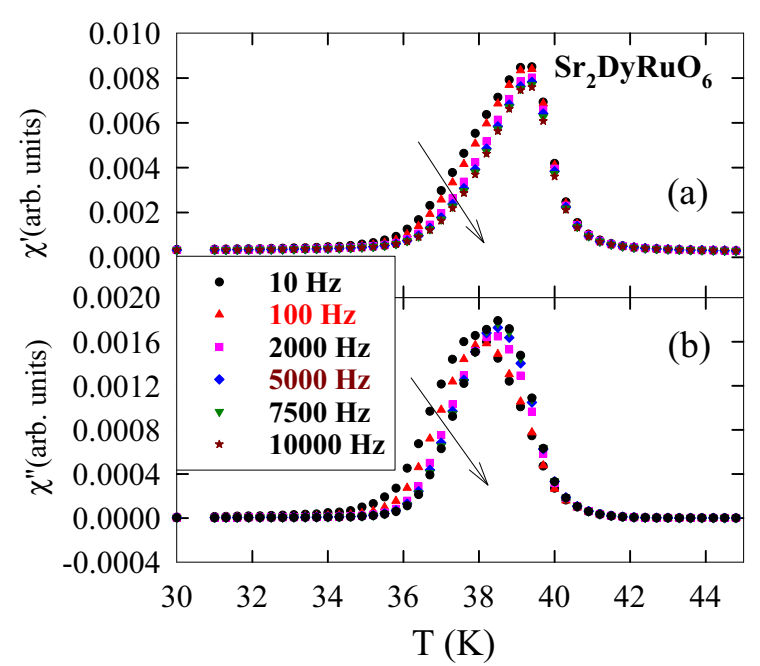

FIG. 5. (a) Real and (b) imaginary components of AC susceptibility $\left(\chi_{\mathrm{AC}}\right)$ measured at various frequencies as a function of temperature.

the contribution of the weak ferromagnetic component from the DM interaction. The magnetization attains a value of only $\sim 4.31 \mu_{\mathrm{B}}$ at $7 \mathrm{~T}$ and it does not exhibit saturation behavior at $7 \mathrm{~T}$ field at $2 \mathrm{~K}$, which is in good agreement with existing literature [41].

Figure 5 represents the real $\chi_{\mathrm{AC}}^{\prime}$ and the imaginary part $\chi_{\mathrm{AC}}^{\prime \prime}$ of the temperature dependent AC susceptibility of SDRO at frequencies ranging from $10 \mathrm{~Hz}$ to $10 \mathrm{kHz}$. Both anomalies as observed in the heat capacity and $\chi_{\mathrm{DC}}$ are visible in the real and imaginary parts. The anomaly at $T_{N 1}=40 \mathrm{~K}$ is revealed by a sharp jump in both $\chi_{\mathrm{AC}}^{\prime}$ and $\chi_{\mathrm{AC}}^{\prime \prime}$, and is frequency independent, whereas the anomaly at $T_{N 2}$ creates a broad shoulder to the main transition and shows a weak frequency dispersion. This indicates that the second ordering is possibly associated with a very small change in the spin structure near $T_{N 2}$.

\section{C. $\mu \mathrm{SR}$ measurements}

In order to gain insight as to whether the two observed phase transitions in the heat capacity originate from the magnetic ordering, we have investigated SDRO using the $\mu \mathrm{SR}$ technique. $\mu \mathrm{SR}$ is a local microscopic probe and is sensitive to extremely small internal fields and is ideal to detect spatially inhomogeneous magnetic features. It is extensively applied to investigate small changes in magnetism [51]. It is interesting to mention that $\mu \mathrm{SR}$ studies on double perovskites have provided important information on the magnetic ground state of these systems [40,52-54], including information on the microscopic coexistence of magnetic and nonmagnetic phases in $\mathrm{Ba}_{2} \mathrm{PrRu}_{9.9} \mathrm{Ir}_{0.1} \mathrm{O}_{6}$ [55]. For the present study, zero field (ZF) and longitudinal field (at constant temperature) $\mu \mathrm{SR}$ measurements have been performed. Figure 6 shows the muon asymmetry versus time spectra at several temperatures between 5 and $90 \mathrm{~K}$ measured in ZF. The analysis of $\mu \mathrm{SR}$ spectra was carried out using a stretched exponential function with constant background:

$$
G_{z}(t)=A_{0} \exp \left[-(\lambda t)^{\beta}\right]+A_{\mathrm{bg}} .
$$




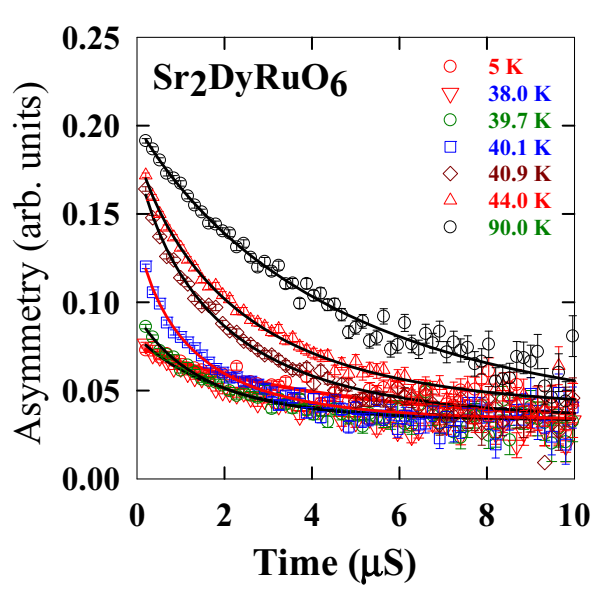

FIG. 6. Zero-field $\mu$ SR spectra measured at various temperatures. The experimental data are shown by the symbols and the solid line shows fit to the data using stretch exponent function.

Here $A_{0}$ is the muon initial asymmetry and $\lambda$ is the muon relaxation rate. If the exponent $\beta=2$, the function becomes Gaussian while for $\beta=1$, the function becomes exponential. $A_{\mathrm{bg}}$ is the constant background arising from muons stopping on the Ag-sample holder. Furthermore, $\beta<1$ describes inhomogeneous dynamic relaxation where the relaxation is locally exponential but the local rates are distributed [56]. It has no basic theoretical justification, but is often used as a convenient characterization of an a priori unknown distribution of relaxation rates. As in SDRO we would expect different internal fields for muons stopping on the $\mathrm{DyO}_{6}$ octahedral site and on the $\mathrm{RuO}_{6}$ octahedral site. The present approach to fitting the data with the use of a stretched exponential function therefore seems appropriate. We have also fitted the $\mu$ SR spectra of SDRO using a simple exponential function (see the Supplemental Material, Figs. S1 and S2 [57]) and the results are very similar to that obtained from a stretched exponential function fit given here. It is to be noted that a simple exponential function fits better to the low temperature and high temperature data, however the fits are not very good for the data close to $T_{N}$.

Figure 7(b) shows that at high temperature (i.e., above the Néel temperature) the $\mu \mathrm{SR}$ spectra exhibit a moderate relaxation rate, which is due to the spin fluctuation from the $\mathrm{Ru}^{5+}$ and $\mathrm{Dy}^{3+}$ moments. With decreasing temperature, the relaxation rate increases and exhibits a peak near $40 \mathrm{~K}$, followed by a rapid loss of muon initial asymmetry [Fig. 7(a)] below $40 \mathrm{~K}$. Between 1.2 and $40 \mathrm{~K}$, the asymmetry loss is almost $2 / 3$. In the polycrystalline sample, we expect three components of internal field at the muon stopping sites. The loss of 2/3 can be understood as implanted muons see three components of the internal field in the polycrystalline sample. One longitudinal component, which is the component of internal field that is parallel to the incident muon beam ( $1 / 3$ component), will give a relaxation and no oscillations. While the remaining two components seen by the muons ( $2 / 3 \mathrm{com}-$ ponent) are the transverse components (i.e., components of the internal field perpendicular to the incident muon beam), which give oscillations. As the internal field from the $\mathrm{Dy}^{3+}$ and $\mathrm{Ru}^{5+}$ moments are expected to be larger and inhomogeneous, the

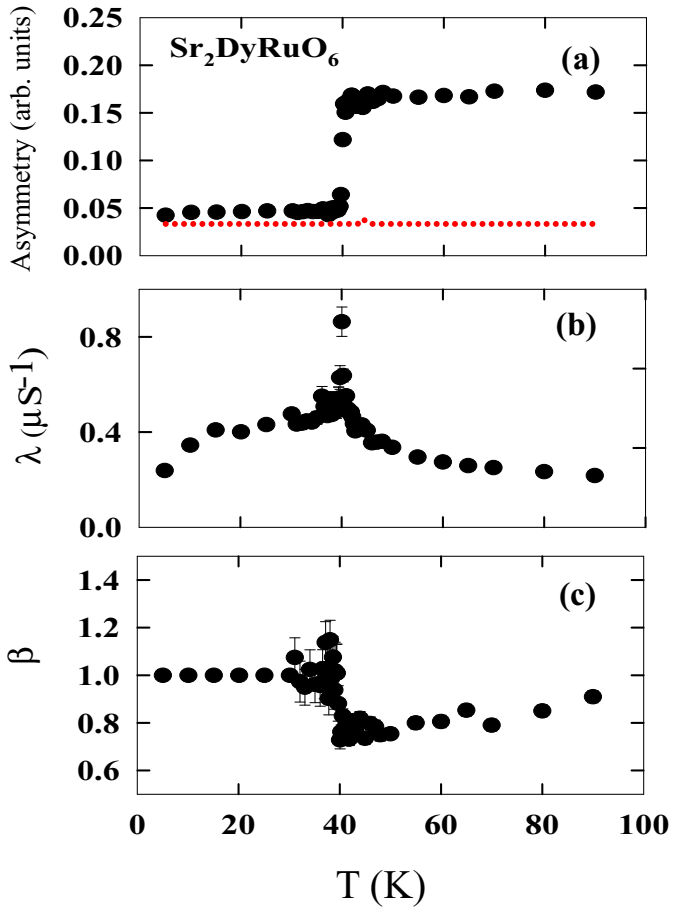

FIG. 7. The temperature dependent fit parameters obtained from the zero-field $\mu$ SR spectra. (a) Initial muon asymmetry versus temperature, please note that the symbols are the sample asymmetry only after subtracting the background asymmetry from the sliver sample holder shown by the dotted line, see Eq. (1). (b) Relaxation rate versus temperature and (c) exponent $\beta$ versus temperature. The dotted line in (a) shows the temperature independent background from the sample holder.

oscillations in the muon time evolution asymmetry will be damped rapidly. Therefore, due to the pulse width of the ISIS muon beam, it is difficult to observe these oscillations in the $2 / 3$ component as this signal damps very quickly in the short time window close to zero time. We therefore attribute the observed jump in $A_{0}$ at $40 \mathrm{~K}$ to long-range magnetic ordering of both $\mathrm{Dy}^{3+}$ and $\mathrm{Ru}^{5+}$ moments, as also observed in the heat capacity and magnetization measurements. This is also in agreement with our finding from the neutron diffraction study discussed below. Furthermore, if we look in detail at the behavior of $\lambda(T)$ near $36 \mathrm{~K}$, then there is weak evidence of a second phase transition in $\lambda(T)$. However, as expected, $A_{0}$ does not reveal any sign of a second transition as the system is already in a complete long-range magnetic ordered state below $40 \mathrm{~K}$ and hence cannot lose further asymmetry. We therefore attribute the weak change in the $\lambda(T)$ near $36 \mathrm{~K}$ as due to a very small change in the spin configuration. The exponent $\beta$ reveals a temperature dependent behavior. It starts to decrease with temperature from $\sim 1$ at $90 \mathrm{~K}$ to $\sim 0.7$ at $40 \mathrm{~K}$ and then exhibits a sharp jump to 1 below $T_{N 1}$ and remains the same to lower temperature. It is interesting to note that a very recent $\mu$ SR study on $\mathrm{La}_{2} \mathrm{NaRuO}_{6}$ and $\mathrm{La}_{2} \mathrm{NaOsO}_{6}$ [40] reports similar features in $\lambda(T)$, while only exhibiting a single magnetic transition. The $\mu \mathrm{SR}$ spectra of these compounds were also better fitted to a stretched exponential function. In SDRO there are no clear signs of frequency oscillations. This indicates that the internal fields at the muon 
stopping sites, which are most likely close to the oxygen ions due to the negative charge on $\mathrm{O}^{2-}$ and positive charge on muon, are larger than $800 \mathrm{G}$. The width limit of the ISIS muon pulse does not allow the collection of data at very short times close to zero. The ordered moment of $\mathrm{Dy}^{3+}$ is quite high compared to $\mathrm{Ru}^{5+}$ (discussed in the neutron diffraction section), and it is highly possible that muons stopping in the $\mathrm{DyO}_{6}$ octahedra will see a higher internal field than those stopping in the $\mathrm{RuO}_{6}$ octahedra. We have also performed LF measurements at 5, 35, and $50 \mathrm{~K}$ up to $2.5 \mathrm{kG}$ field (see Fig. S3 of the Supplemental Material (SM) [57]). At 5 and $35 \mathrm{~K}$ the initial asymmetry gradually recovers to 0.12 and 0.15 , respectively, at $2.5 \mathrm{kG}$ field. Furthermore, the relaxation rate also exhibits a gradual decrease with applied field. On the other hand, at $50 \mathrm{~K}$ the initial asymmetry does not change much with applied field, but the relaxation rate decreases slowly with applied field.

\section{Neutron powder diffraction studies}

To explore the origin of the two anomalies observed in various experiments, temperature dependent NPD measurements have been performed on the high intensity diffractometer D20 between 1.7 and $50 \mathrm{~K}$ with $\lambda=1.594 \AA$ (Fig. 8). Long scans of $45 \mathrm{~min}$ have been performed at 1.7, 10, 20,30, 40 and $50 \mathrm{~K}$ and shorter scans of $\sim 11$ min were recorded between these temperatures while raising the temperature with a constant ramp of $1 \mathrm{~K} / 5 \mathrm{~min}$. Figure 8(a) represents the NPD patterns at 1.7 and $50 \mathrm{~K}$, along with the difference curve plotted in green. Several new peaks emerge and some existing ones increase in intensity at low temperature indicating the onset of long-range magnetic ordering. The magnetic reflections can be indexed with $h+k+l$ being odd. Figure 8(b) is the 3D plot of the temperature dependent D20 data in a limited $2 \theta$ range in which the magnetic peaks are indexed using the nuclear unit cell, i.e., the magnetic propagation vector $k=(0,0,0)$. This is consistent with $A$-type antiferromagnetic order where ferromagnetic sheets within the $a b$ plane are antiferromagnetically stacked along the long $c$ axis with both the magnetic and crystal unit cell of same size. The presence of the (001) reflection indicates that the magnetic moments have components in the $a b$ plane. The strongest magnetic peak represents in fact two reflections which can be indexed as $(010) /(100)$, but cannot be resolved due to the $a \sim b$ pseudosymmetry. Magnetic symmetry analysis for the two possible magnetic sites of $\mathrm{Dy}^{3+}$ on $2 c$ and $\mathrm{Ru}^{5+}$ on $2 d$ was done in space group $P 2_{1} / n$ with $k=(0,0,0)$ using the program BASIREPS $[58,59]$. Both sites possess the same two allowed irreducible representations (IR) with each having three basis vectors (BV) (Table I). The two IRs are differing in the directions of the ferromagnetic $(\mathrm{F})$ or antiferromagnetic (AF) couplings between the symmetry related sites within the unit cell. While IR1 allows an AF coupling along the unit cell $b$ direction with $\mathrm{F}$ couplings allowed along $a$ and $c$ directions. The IR2 describes just the opposite with a F coupling along $b$ and AF couplings along $a$ and $c$. Testing both IRs, it becomes clear that due to the above mentioned pseudosymmetry, it is possible to refine the low temperature magnetic structure model assuming either an AF coupling along $a$ (IR1) or along $b$ (IR2). In both cases a single $\mathrm{BV}$ is sufficient to

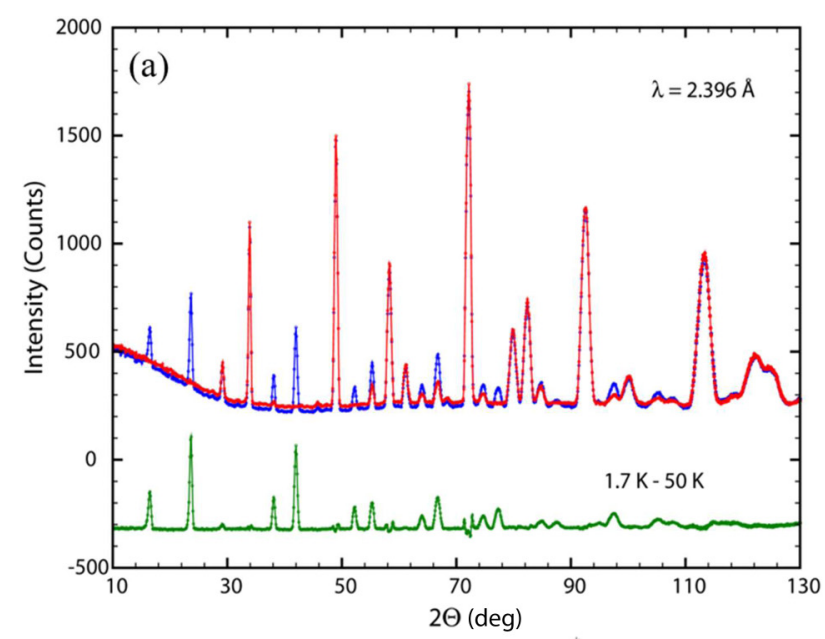

(b)

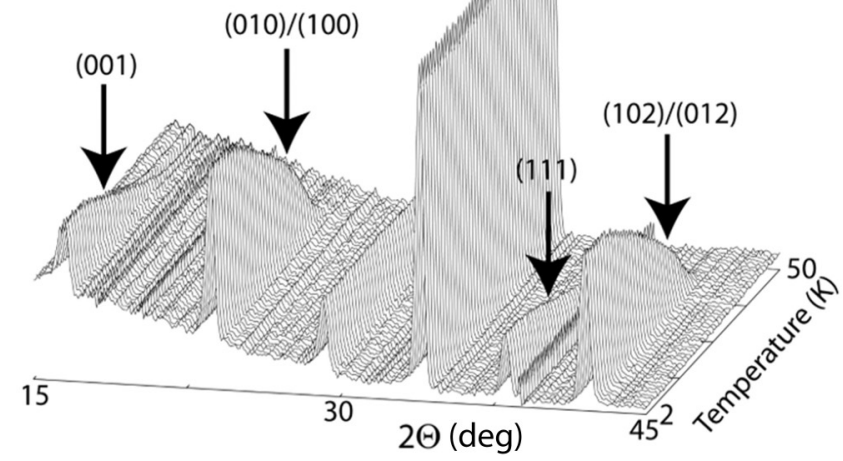

FIG. 8. Neutron diffraction pattern at 1.7 (blue curve) and $50 \mathrm{~K}$ (red curve) measured using D20. At $1.7 \mathrm{~K}$, extra peaks are present compared to $50 \mathrm{~K}$, which is due to magnetic ordering of the $\mathrm{Dy}^{3+}$ and $\mathrm{Ru}^{5+}$ moments. The green line at the bottom represents the difference curve $(1.7-50 \mathrm{~K})$ and shows only the magnetic Bragg peaks. (b) Thermal evolution of magnetic peak profiles between 2 and $50 \mathrm{~K}$. The arrows show the magnetic Bragg peaks.

refine the magnetic structure, there is no clear indication of a ferromagnetic contribution to the magnetic scattering. As even our high resolution data from D2B at $3 \mathrm{~K}$ using $\lambda=1.594 \AA$ are not able to differentiate between the two models [Fig. 9(b)], additional NPD data have been collected at $3.5 \mathrm{~K}$ as well on the high-resolution powder diffractometer D2B using the longer wavelength of $\lambda=2.399 \AA$ in order to determine whether the moments are pointing in the $a$ or $b$ directions (Fig. 10). The only magnetic peak which allows us to differentiate between these two possibilities is the $(100) /(010)$ doublet. Figure 10 shows the Rietveld refined NPD pattern measured at $3.5 \mathrm{~K}$. The insert shows the enlarged view of the $(010) /(100)$ peaks. It clearly shows that there is magnetic intensity at the (100) reflection but not on the (010) reflection, which confirms that the magnetic moments are pointing in the $b$ direction. The resultant magnetic structure is shown in Fig. 11 in which the $\mathrm{Ru}^{5+}$ moments are represented in green color and the $\mathrm{Dy}^{3+}$ moments in red color. Using this model, the magnetic structure was refined using the high resolution data collected on $\mathrm{D} 2 \mathrm{~B}$ at $3 \mathrm{~K}$ and $\lambda=1.595 \AA$, with the magnetic intensity modeled as a second phase containing 


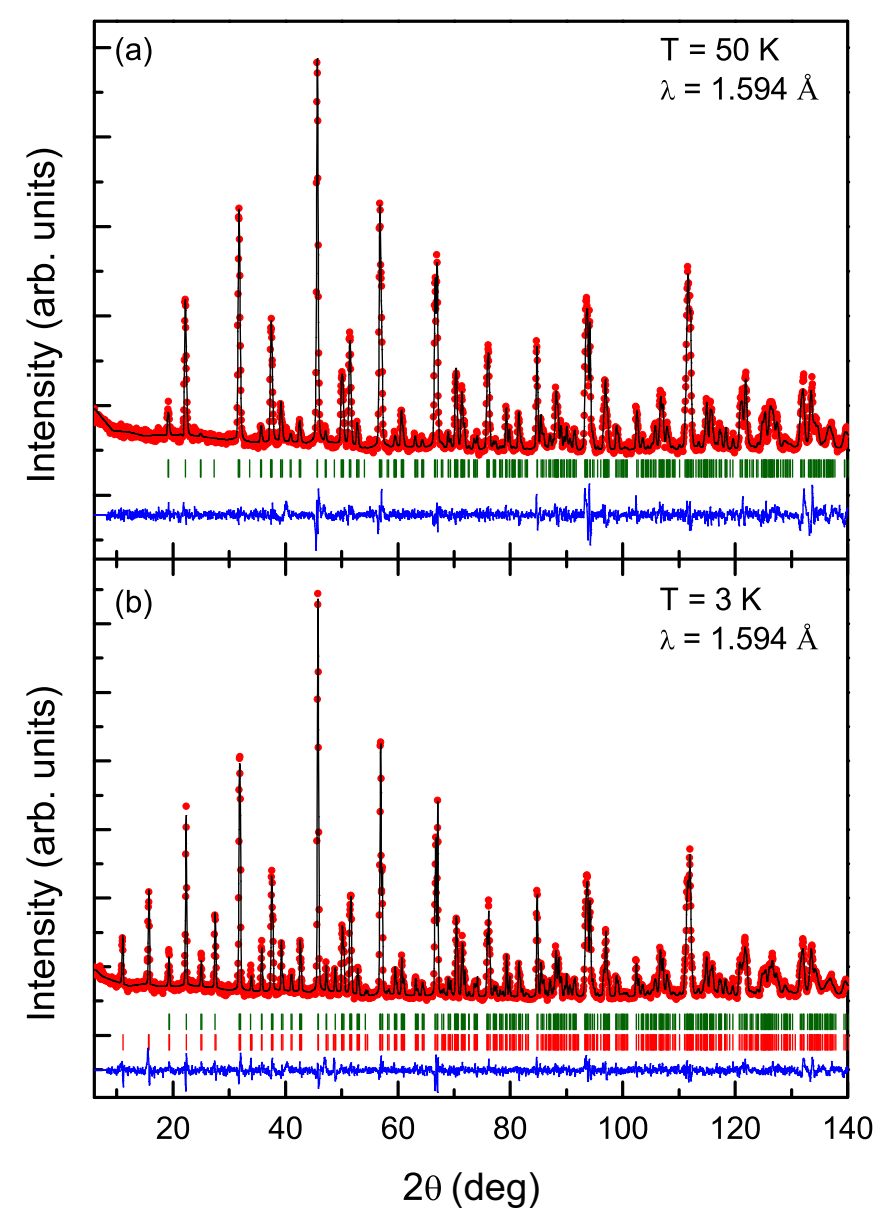

FIG. 9. Rietveld fit to NPD patterns collected at (a) 50 and (b) $3 \mathrm{~K}$ with $\lambda=1.594 \AA$ on D2B diffractometer at ILL: Red circles show the observed data points; the black line the calculated profile; the lower blue line the difference profile (obs. - calc.); upper green vertical markers indicate nuclear Bragg peak positions; lower red vertical markers in (b) indicate magnetic Bragg peak positions.

only the Dy and Ru atoms. The magnetic form factor used for $\mathrm{Ru}^{5+}$ is the one determined empirically in [60]. Figure 9(b) shows this refinement where the lower set of red tick marks correspond to the magnetic Bragg reflections. Table II shows the relevant bond lengths and angles at $T=3 \mathrm{~K}$ together with those determined at $T=50 \mathrm{~K}$ [Fig. 9(a)]: Cooling through the magnetic transition leads to a compression of the $\mathrm{RuO}_{6}$ octahedra, whereas the $\mathrm{DyO}_{6}$ octahedra elongate.

A cyclic structure refinement using the temperature dependent data from D20 allowed the determination of the thermal dependence of the magnetic moments of $\mathrm{Dy}^{3+}$ and $\mathrm{Ru}^{5+}$ and is shown in Fig. 12(a) with the normalized moments plotted in Fig. 12(b). It can be seen that for the present system, the $\mathrm{Ru}^{5+}$ moment attains saturation at a faster rate near $\sim 20 \mathrm{~K}$ compared to $\mathrm{Dy}^{3+}$ which attains saturation only well below $\sim 10 \mathrm{~K}$. This behavior is similar to other members of this family, like $\mathrm{Sr}_{2} \mathrm{HoRuO}_{6}$ and $\mathrm{Sr}_{2} \mathrm{TbRuO}_{6}$ [38]. However, the direction of the magnetic moments of $\mathrm{Dy}^{3+}$ and $\mathrm{Ru}^{5+}$ are different in the present system from those of the Ho and Tb based double ruthenates. While both the rare earth and the $\mathrm{Ru}^{5+}$ moments are along the $c$ axis in $\mathrm{Sr}_{2} \mathrm{HoRuO}_{6}$, they are canted

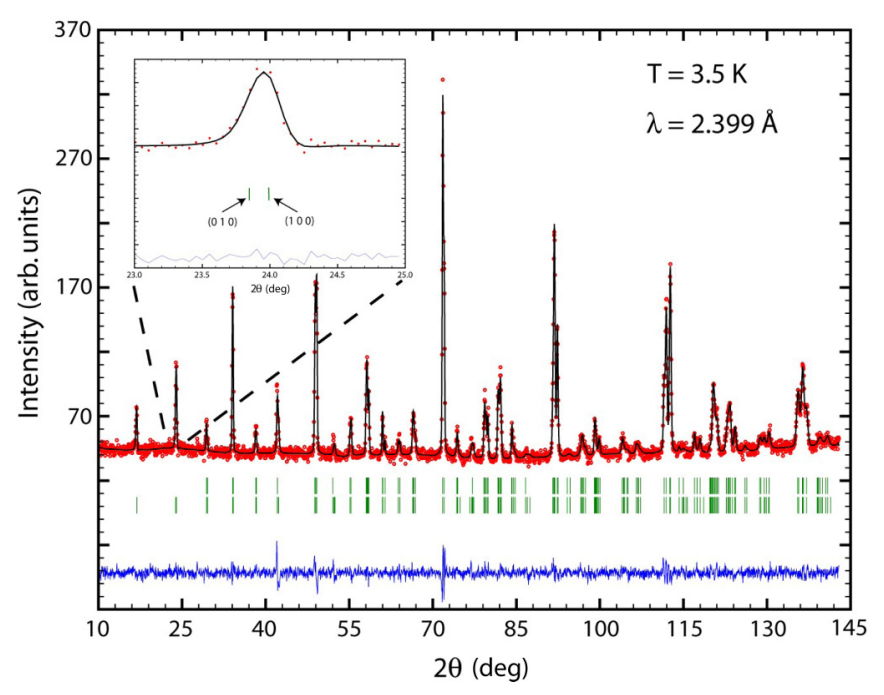

FIG. 10. Rietveld fit to NPD patterns collected at $3.5 \mathrm{~K}$ with $\lambda=2.399 \AA$ on D2B diffractometer at ILL: Red circles show the observed data points; the black line the calculated profile; the lower blue line the difference profile (obs. - calc.); upper green vertical markers indicate nuclear Bragg peak positions; lower red vertical markers indicate magnetic Bragg peak positions. The inset enlarges the view near $24^{\circ}$ to highlight the magnetic peak (010)/(100).

by $20^{\circ}$ from the $c$ axis for $\mathrm{Sr}_{2} \mathrm{TbRuO}_{6}$ [38]. The moments of $\mathrm{Ru}$ and $\mathrm{Pr}$ in the $a c$ plane (i.e., tilted away from the $c$ axis) were also found in $\mathrm{Ba}_{2} \mathrm{PrRuO}_{6}$ [55]. Furthermore, the magnetic structure of $\mathrm{Sr}_{2} \mathrm{ErRuO}_{6}$ shows $\mathrm{Ru}^{5+}$ and $\mathrm{Er}^{3+}$ moments are mainly aligned along the $c$ axis of the structure, forming an angle of $\sim 6^{\circ}$ with the $c$ axis in the case of the $\mathrm{Er}^{3+}$ sublattice and $\sim 15^{\circ}$ for the $\mathrm{Ru}^{5+}$ moment [61]. In the present studied system, both the $\mathrm{Dy}^{3+}$ and $\mathrm{Ru}^{5+}$ moments are pointing along the $b$ axis. The values of the $\mathrm{Dy}^{3+}$ and the $\mathrm{Ru}^{5+}$ moments at $1.7 \mathrm{~K}$ are $\mu_{\mathrm{Dy}^{3+}}=4.92(10) \mu_{\mathrm{B}}$ and $\mu_{\mathrm{Ru}+5}=1.94(7) \mu_{\mathrm{B}}$. The strong reduction of $\mu_{\mathrm{Dy} 3+}$ compared to the expected value

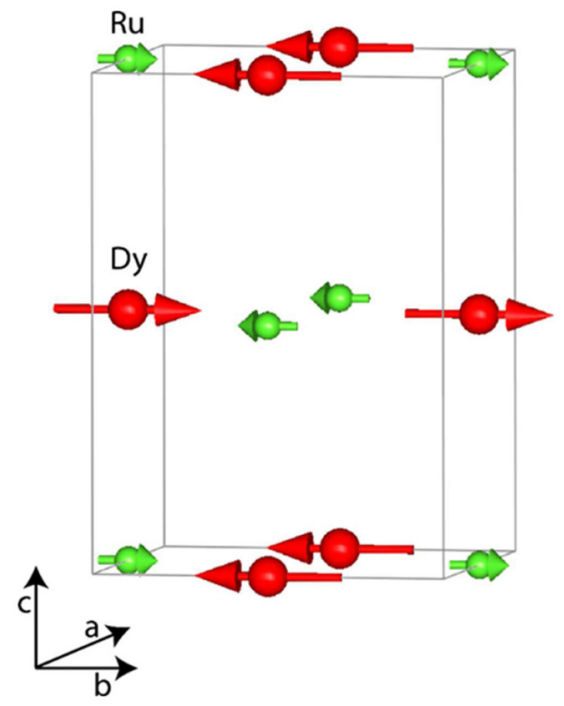

FIG. 11. The magnetic structure of $\mathrm{Sr}_{2} \mathrm{DyRuO}_{6}$ for $k=(0,0,0)$. The $\mathrm{Dy}^{3+}$ and $\mathrm{Ru}^{5+}$ moments are shown in red and green colors, respectively. 
TABLE I. Basis vectors (BV) of the allowed irreducible representations (IR) for $k=(0,0,0)$ for the Wykoff positions $2 c$ (Dy) and $2 e(\mathrm{Ru})$ of the space group $P 2_{1} / n$.

\begin{tabular}{lrcc}
\hline \hline IR1 & BV1 & BV2 & BV3 \\
\hline$x, y, z$ & 100 & 010 & 001 \\
$-x+1 / 2, y+1 / 2,-z+1 / 2$ & -100 & 010 & $00-1$ \\
IR2 & & & \\
$x, y, z$ & 100 & 010 & 001 \\
$-x+1 / 2, y+1 / 2,-z+1 / 2$ & 100 & $0-10$ & 001 \\
\hline \hline
\end{tabular}

of $10 \mu_{\mathrm{B}}$ in line with similar discrepancies observed for the other rare earth Ru-based perovskites [38]. The $\mathrm{Ru}^{5+}$ moment value is similar to those reported for other members of this family $[36,38,46]$ and points to the fact that in these systems the super-super exchange $\mathrm{Ru}-\mathrm{O}-\mathrm{O}-\mathrm{Ru}$ interactions are the strongest magnetic interactions and control the $\mathrm{Ru}$ ordering. The similar values of $\mu_{\mathrm{Ru}^{5+}}$ and of the magnetic transition temperatures in the different $\mathrm{Sr}_{2} \mathrm{LnRuO}_{6}$ systems [38] are explained by the weakness of the $\mathrm{Ln}$-O-Ru interactions. As exemplified by the very low magnetic transition temperature of $\mathrm{Dy}_{2} \mathrm{O}_{3}\left(T_{N}=1.2 \mathrm{~K}\right)$, Dy-O-Dy interactions are in general very weak. In the well-ordered double perovskite SDRO, only weaker super-super exchange Dy-O-O-Dy interactions are present which cannot be the origin of the primary ordering of $\mathrm{Dy}^{3+}$ at $39 \mathrm{~K}$ as supported by the order parameters given in Fig. 12(b). The temperature dependence of the $\mathrm{Ru}^{5+}$ moments exhibits a mean field power-law behavior with a critical exponent $\beta=0.56(1)$, whereas that of the $\mathrm{Dy}^{3+}$ moments deviates from the power law. Hence, it appears that $\mathrm{Ru}^{5+}$ induces the rare earth ordering in these systems leading to a simultaneous ordering of $\mathrm{Dy}^{3+}$ at the same temperature, as also previously reported for $\mathrm{Sr}_{2} \mathrm{LnRuO}_{6}$ ( $\mathrm{Ln}=\mathrm{Ho}$ and $\mathrm{Tb}$ ) [38] as well as in $\mathrm{R}_{2} \mathrm{RuO}_{5}$ [62-64]. It should be noted that there appear neither appreciable changes in the magnetic peak profiles nor new magnetic Bragg peaks in the temperature dependent data across the second anomaly $(\sim 36 \mathrm{~K})$. In particular we did not detect any additional superlattice peak in the temperature region between $T_{N 1}$ and $T_{N 2}$, such as the one created by a propagation vector $k=(1 / 2,1 / 2,0)$ found by Bernardo et al.
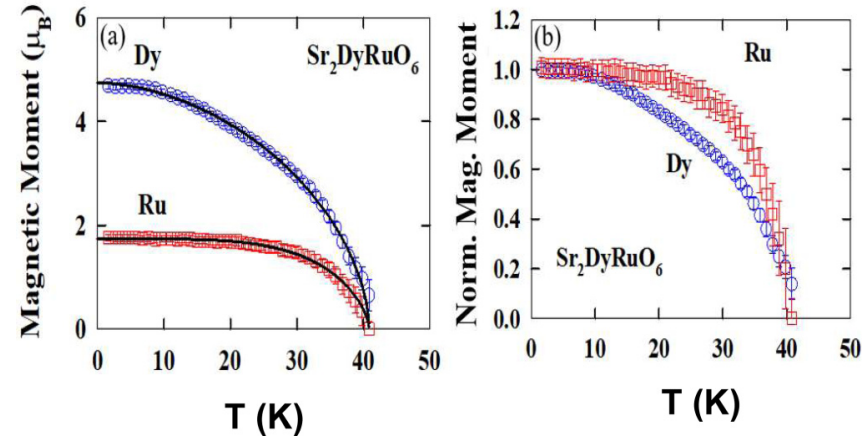

FIG. 12. Thermal variation of (a) $\mathrm{Dy}^{3+}$ and $\mathrm{Ru}^{5+}$ moments and (b) normalized moments of $\mathrm{Dy}^{3+}$ and $\mathrm{Ru}^{5+}$.

[35] in $\mathrm{Sr}_{2} \mathrm{YRuO}_{6}$. Furthermore, there is no indication for short-range correlations in the background below $T_{N 1}=40 \mathrm{~K}$. This indicates that the second transition might be associated with very small changes in the spin structure/spin reorientation which are beyond the detection limit even of the high intensity data. Although we are not able to detect any clear changes in the temperature dependent neutron data at $T_{N 2}$, we tested the idea that a spin reorientation could be linked to the appearance of a symmetry allowed ferromagnetic component. Trying to include such a component in the refinement of the $1.7 \mathrm{~K}$ high intensity data, values of $0.8(5) \mu_{\mathrm{B}}$ for the Dy site or $1.0(0.7) \mu_{\mathrm{B}}$ for the Ru site were determined. Allowing a simultaneous presence of ferromagnetic components on both sites leads to the divergence of the refinements. Hence, and looking at the very large error bars, we can neither claim the presence of a ferromagnetic component nor its localization. Refinement of the long scan taken at $30 \mathrm{~K}$ including ferromagnetic components leads to even less precise values of about $0.4(0.8) \mu_{\mathrm{B}}$ on either site. We therefore cannot ascertain but only mention that the second transition could be caused by the appearance of a ferromagnetic component leading to a spin reorientation. This ferromagnetic component would then be as well responsible for the weak ferromagnetic hysteresis observed in the magnetization isotherm at low temperature.

The possible magnetic frustration present in double perovskites is a consequence of the crystal structure as both $B$

TABLE II. Selected bond lengths ( $)$ ) and bond angles (deg) across AFM ordering.

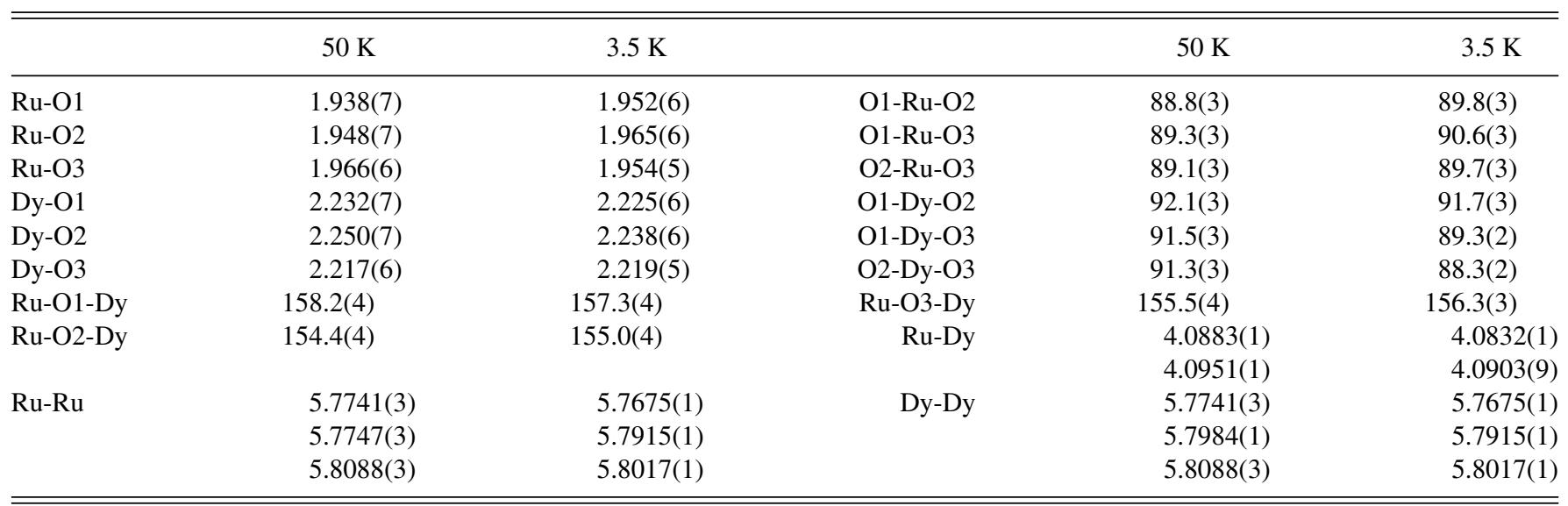


sites form lattices of edge-sharing tetrahedra resembling the arrangement in a face centered cubic lattice. Nearest neighbor $(\mathrm{NN})$ distances within one tetrahedron and to the neighboring tetrahedra are very similar so that any antiferromagnetic interaction must lead to a situation where conflicting demands on the orientations of equidistant neighboring spins arise. This is especially true when there is only one magnetic sublattice present, i.e., as in $\mathrm{Sr}_{2} \mathrm{YRuO}_{6}$, which can lead to magnetic structures reflecting inherent frustration [36]. In SDRO two magnetic sublattices are present, one from $\mathrm{Ru}$ and another from Dy. If the intersublattice interactions Dy-O-Ru via superexchange are important, the frustration created through the intrasublattice interactions (i.e., between $\mathrm{Ru}-\mathrm{Ru}$ and Dy-Dy) can be overcome and the system can order in a long-range magnetic state. The importance of the intersublattice interaction is in the case of SDRO reflected in the fact that both sublattices order at the same temperature (see Fig. 12) and with the same magnetic propagation vector. Intersublattice superexchange interactions of very similar distances and $\mathrm{Ru}-\mathrm{O}-$ Dy angles are found along the $c$ direction of the unit cell and within the $a-b$ plane (Fig. 11). There are, however, four RuO-Dy NN pathways within the $a-b$ plane while there are only two along the $c$ direction. Assuming that these superexchange interactions are of antiferromagnetic type, the magnetic structure adopted in SDRO (Fig. 11) shows that the interactions within the $a-b$ plane are dominant. Keeping in mind that the intrasublattice $\mathrm{NN} \mathrm{Ru}-\mathrm{O}-\mathrm{Ru}$ superexchange interactions are still the strongest of all interactions-determining the temperature of the magnetic transition $T_{N 1}$, see above-an antiferromagnetic alignment between $\mathrm{Ru}$ sites in neighboring $a-b$ layers is established. This in turn forces a ferromagnetic alignment between Ru and Dy sites in neighboring $a-b$ layers. The magnetic structure adopted sees therefore two antiferromagnetic and two ferromagnetic $\mathrm{NN}$ intrasublattice interactions for both the Ru and the Dy tetrahedra. As the monoclinic distortion is very small the intrasublattice interaction distances and angles are, however, very similar and one has to conclude that some frustration should still be present in the compound. This is neither visible through the presence of some static diffuse scattering in the neutron diffraction data nor in the frustration index $f=\left|\Theta_{\mathrm{CW}}\right| / T_{N} \sim 0.7$ but could be reflected in the low values of the magnetic moment of $\mathrm{Dy}^{3+}=4.9(1) \mu_{\mathrm{B}}$ and even $\mathrm{Ru}^{5+}=1.94(7) \mu_{\mathrm{B}}$.

In the case of SDRO the two magnetic transitions observed in the heat capacity are not discernible in the neutron diffraction patterns as a change of the magnetic structure across the two transitions. This is not uncommon as there are several materials where heat capacity shows two (or three) peaks, but neutron diffraction data do not show clear visible changes between these transitions [65-67]. In the case of CeIrGe 3 the neutron diffraction measurements could only detect a very small change in the magnetic propagation vector from $k=(0,0,0.6667)$ to $\left(\begin{array}{lll}0 & 0 & 0.688\end{array}\right)$ across the three transitions defined through three peaks in the heat capacity [65]. A second example concerns the multiferroic compound $\mathrm{Mn}_{3} \mathrm{TeO}_{6}$ exhibiting two peaks in the heat capacity at $T_{N 1}=23 \mathrm{~K}$ and at $T *=T_{N 2}=21 \mathrm{~K}$, temperature below which a multiferroic state emerges. The neutron diffraction study on $\mathrm{Mn}_{3} \mathrm{TeO}_{6}$ reveals only one magnetic transition at $24 \mathrm{~K}$ and the same incommensurately modulated magnetic spin structure with the propagation vector $k=(0,0,0.43)$ is kept in the whole temperature range from 5 to $24 \mathrm{~K}$ [66]. A third example is $\mathrm{YbRu}_{2} \mathrm{Ge}_{2}$ [67] where the heat capacity shows two magnetic transitions, $T_{N 1}=6.5 \mathrm{~K}$ and $T_{N 2}=5.5 \mathrm{~K}$. Neutron data revealed here only a tiny change in the magnetic propagation vector [from $\mathbf{k}=(0.35200)$ to $(0.35000)$ ] without any indication of this transition in the intensity of the magnetic reflections. These examples show that the presence of a peak in the heat capacity data does not necessarily lead to a detectable change in the neutron data and the first and third examples show that tiny changes in the configuration can induce peaks in the specific heat data. In the case of $\mathrm{Sr}_{2} \mathrm{DyRuO}_{6}$ treated here, we can associate the first peak to the appearance of the antiferromagnetic order. The second peak in the heat capacity of $\mathrm{Sr}_{2} \mathrm{DyRuO}_{6}$ could be related to the appearance of additional ferromagnetic interactions leading to the spin reorientation.

\section{E. Inelastic neutron scattering studies}

It is very important to understand the origin of the reduced magnetic moment and the strength of anisotropy in SDRO. We therefore have performed inelastic neutron scattering (INS) measurements on SDRO at various temperatures. Figures 13(a)-13(d) show the color contour maps of the scattering intensity, energy transfer vs momentum transfer $(Q)$, at various temperatures between 7 and $45 \mathrm{~K}$ with a neutron incident energy $E_{i}=15 \mathrm{meV}$. At $7 \mathrm{~K}$ a strong band of excitation can be seen near $3.25 \mathrm{meV}$, and weak scattering intensity near $5.63,6.8$, and $8.9 \mathrm{meV}$, which are more clear when presented in 1D intensity versus energy plots between $Q=0$ and $2 \AA^{-1}$ [Figs. 13(e) and 13(f)]. At $30 \mathrm{~K}$, the $3.25 \mathrm{meV}$ excitation softens and it seems scattering intensity emerges out from $Q=1.1 \AA^{-1}$, which is the magnetic Bragg peak with index $\left(\begin{array}{lll}0 & 1 & 0\end{array}\right) /\left(\begin{array}{lll}1 & 0 & 0\end{array}\right)$. Increasing the temperature to $37 \mathrm{~K}$, the inelastic scattering broadens and transforms into diffuse scattering with an energy width of $\sim 8 \mathrm{meV}$. At 45 and $55 \mathrm{~K}$ (above $T_{N}$ ), we have seen the presence of weak and broad diffuse scattering in the elastic cut (see Fig. S4 in the SM [57]), which suggests the presence of magnetic frustration/short-range correlations above $T_{N}$ in SDRO. As we did not see a clear sign of diffuse scattering in the diffraction data on D20, it may suggest that the diffuse scattering has a dynamic nature. From the data at $5 \mathrm{~K}$, we confirm the presence of a spin wave at $7 \mathrm{~K}$ with a spin gap of $\sim 3.25 \mathrm{meV}$ and zone boundary energy of $8.9 \mathrm{meV}$. It is to be noted that the observed scattering could be also partly interpreted as Zeeman splitting of the Dy low energy crystal field excitation (CEF) levels below $T_{N}$. As there are in total four magnetic atoms per unit cell (2 Dy and $2 \mathrm{Ru}$ ) one expects four spin wave branches, which is in agreement with the observed four excitations below $10 \mathrm{meV}$ [Figs. 13(e) and 13(f)]. Next we compare the value of the spin gap (defined as the peak position in the energy cut, $q$ integrated close to the AFM zone center for the powder samples) observed in the present system with those reported in other double perovskites systems [68-71]. Our INS on $\mathrm{Sr}_{2} \mathrm{YRuO}_{6}$ reveals a spin gap of $5 \mathrm{meV}$ [68], which agrees with the reported results [72]. Spin gaps of 1.8(8) and 6(1) $\mathrm{meV}$ have been observed in $\mathrm{La}_{2} \mathrm{LiRuO}_{6}$ and $\mathrm{La}_{2} \mathrm{LiOsO}_{6}$, respectively [69], 2.57(4) $\mathrm{meV}$ in $\mathrm{La}_{2} \mathrm{MgIrO}_{6}, 2.09$ (3) in $\mathrm{La}_{2} \mathrm{ZnIrO}_{6}$ [70], 

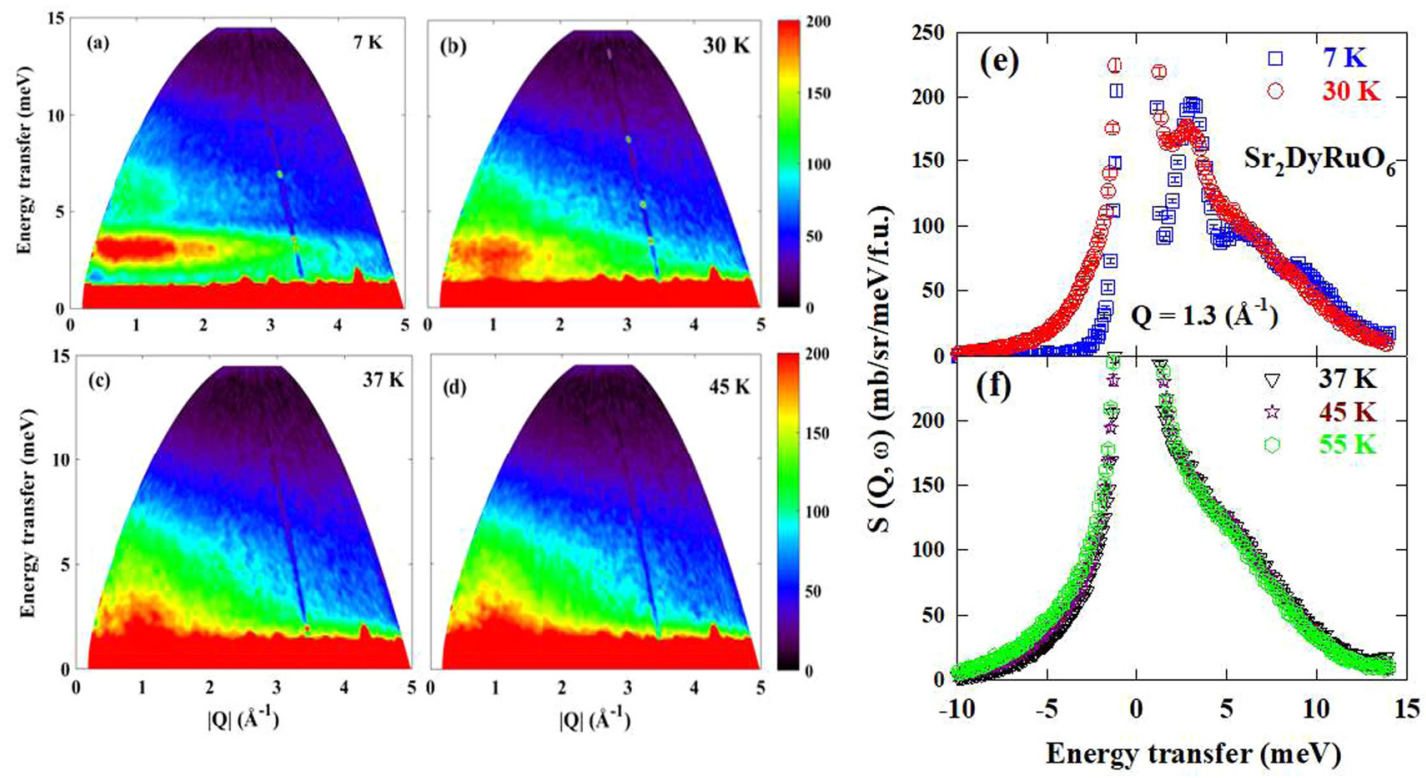

FIG. 13. (a)-(d) The color contour maps of scattering intensity versus momentum transfer at various temperatures measured with an incident energy of $E_{i}=15 \mathrm{meV}$ on MERLIN. (e) and (f) The $Q$-integrated ( $Q=0$ to $2 \AA^{-1}$ ) energy cuts at various temperatures between 7 and $55 \mathrm{~K}$.

$5 \mathrm{meV}$ in $\mathrm{Ba}_{2} \mathrm{YRuO}_{6}$ [71], $2.75 \mathrm{meV}$ in $\mathrm{La}_{2} \mathrm{NaRuO}_{6}$ [40], and $19 \mathrm{meV}$ in $\mathrm{Sr}_{2} \mathrm{ScOsO}_{6}$ [73]. These results may suggest that the spin gap arises from the transition metal $d$ electrons having strong spin-orbital coupling.

Now we discuss the crystal field excitations measured using $E_{i}=130 \mathrm{meV}\left(E_{i}=250 \mathrm{meV}\right.$ data are given in Fig. S6 of the SM). Figures 14(a) and 14(b) shows the color contour maps of the scattering intensity, energy transfer versus momentum transfer, at 7 and $100 \mathrm{~K}$ and Figs. 14(c)-14(e) shows the $Q$-integrated energy cuts from low- $Q$, medium- $Q$, and high- $Q$ data. At $7 \mathrm{~K}$ and at low $Q$, strong intensity of scattering is observed near 46.6 and $73.8 \mathrm{meV}$. Furthermore,
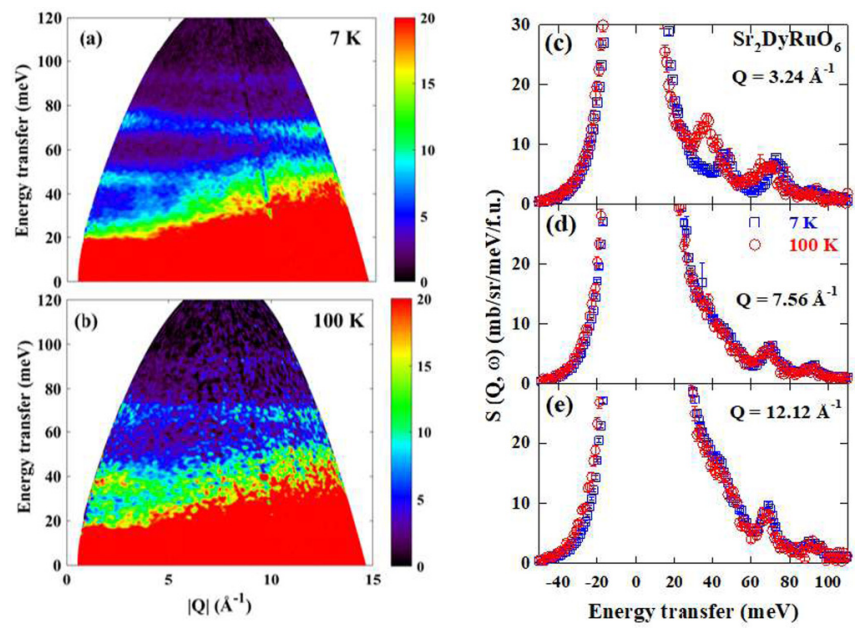

FIG. 14. (a) and (b) The color contour maps of scattering intensity versus momentum transfer at various temperature measured with an incident energy of $E_{i}=130 \mathrm{meV}$ at 7 and $100 \mathrm{~K}$ on MERLIN. (c)-(f) The $Q$-integrated energy cuts at low $Q$, medium $Q$, and at high $Q$ at 7 and $100 \mathrm{~K}$. a weak peak can be seen near $90.8 \mathrm{meV}$ at higher $Q$, which has a lower intensity at lower $Q$. Plotting the $Q$ dependence of the energy-integrated intensity of these peaks (see Fig. S5 in the SM [57]) the intensity of the peaks at 46.6 and $73.8 \mathrm{meV}$ decreases initially and starts to increase at higher $Q$, while that of the $90.7 \mathrm{meV}$ peak increases with $Q$. Moreover, the intensity of the $90.7 \mathrm{meV}$ peak follows a $Q^{2}$ behavior [see the inset in Fig. S5(b)] as expected for phonon scattering. These observations indicate that the 46.6 and $73.8 \mathrm{meV}$ peaks at low $Q$ are due to the crystal field excitations of $\mathrm{Dy}^{3+}$ ion, while the $90.7 \mathrm{meV}$ peak is due to purely phonon scattering. The increase of the intensity of the 46.3 and $73.8 \mathrm{meV}$ peaks at higher $Q$ indicates that phonon modes are present at similar energies suggesting the presence of phonon and CEF coupling. The assignment of CEF and phonon peaks seen in $130 \mathrm{meV}$ data was also confirmed through the measurements with $E_{i}=250 \mathrm{meV}$ at 7 and $120 \mathrm{~K}$ (see Fig. S6 of the SM [57]).

Looking at the data taken at $100 \mathrm{~K}$ with an incoming energy of $130 \mathrm{meV}(250 \mathrm{meV}$ at $120 \mathrm{~K})$ it becomes clear that a new strong peak near $37.3 \mathrm{meV}$ appears (same in $250 \mathrm{meV}$ data at $120 \mathrm{~K}$ ) and that the $73.8 \mathrm{meV}$ peak has shifted to lower energy. It seems that the $46.6 \mathrm{meV}$ peak has also shifted to lower energy. We attribute this new observed peak near $37.3 \mathrm{meV}$ to the excited state transition from the CEF levels below $10 \mathrm{meV}$, which become populated at $100 \mathrm{~K}$. The shift in the peaks could be due to various origins, i.e., magnetoelastic coupling and the Zeeman field at $7 \mathrm{~K}$ from the ordered state moments of the Dy and Ru.

We now discuss the CEF splitting of the $\mathrm{Dy}^{3+}\left(4 f^{9}\right)$ ions based on the CEF Hamiltonian in order to provide further support for our interpretation of the INS spectra. The point symmetry of the $\mathrm{Dy}^{3+}$ ions is triclinic $(1$ or $\mathrm{Ci})$ in the monoclinic $P 2_{1} / c$ crystal structure of SDRO. In such a low symmetry, the CEF Hamiltonian requires 15 CEF parameters 


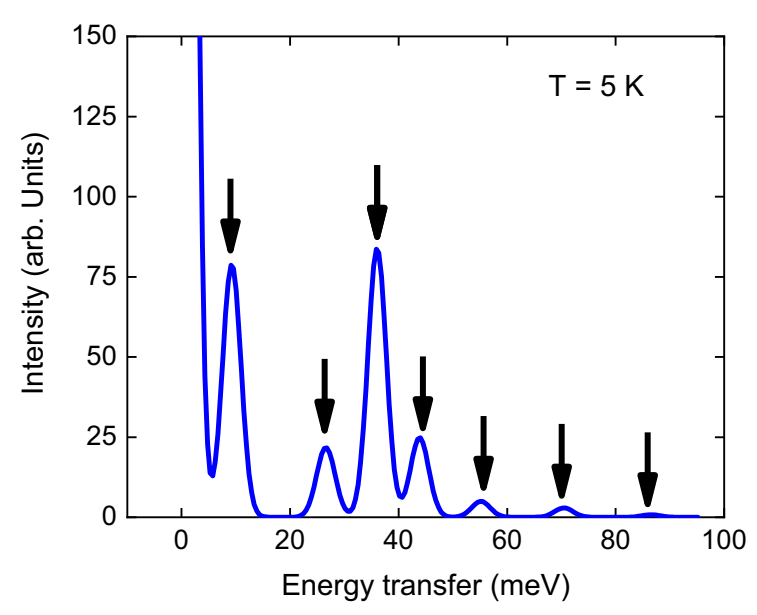

FIG. 15. Crystal field excitations calculated using a point change model with positive sign of $B_{2}{ }^{0}$ in Mantid-crystal field program [75] at $7 \mathrm{~K}$. The linewidth of the excitations was taken as $4 \mathrm{meV}$. Seven crystal field excitations (doubly degenerate) can be seen. The overall splitting qualitatively agrees with the experimental data.

to be estimated from the INS spectra which is a difficult task. Considering an odd number of electrons, $4 f^{9}$ of Dy ${ }^{3+}$ ions and Kramers' theorem [54], which says that for an odd number of electrons, the minimum degeneracy of CEF levels should be twofold (or doubly degenerate) in the paramagnetic state. We therefore expect that the $J=15 / 2$ ground state (16-fold degeneracy $=2 J+1$ ) should split into $8 \mathrm{CEF}$ levels with twofold degeneracy of each level above $T_{N}$. Furthermore, below the magnetic ordering these 8 doublets will split into 16 singlets. Hence if all CEF excitations from the ground state are allowed then in the paramagnetic state, we should expect a minimum of $7 \mathrm{CEF}$ excitations from the ground state. In addition we will have additional CEF excitations due to the excited state transitions at $T=100 \mathrm{~K}>T_{N}$, if there exists low energy CEF levels and the excited state transitions are allowed. Given that we have observed only two CEF transitions at 46.6 and $73.8 \mathrm{meV}$ (in addition to four spin wave type excitations below $9 \mathrm{meV}$ ) at $T=7 \mathrm{~K}$ and one additional excited state transition at $37.3 \mathrm{meV}$ at $100 \mathrm{~K}$ a quantitative analysis of the INS data based on CEF model is not feasible. We have therefore used a point charge model to estimate the $15 \mathrm{CEF}$ parameters (including 12 complex parameters this gives a total of 27 CEF parameters, see Table I in the SM [6]) of the CEF Hamiltonian and calculated INS spectra (Fig. 15) based on these estimated parameters. The simulated INS CEF spectrum at $7 \mathrm{~K}$ is given in Fig. 15. It is to be noted that the point charge model gave a negative sign of $B_{2}{ }^{0}$, corresponding to the $c$ axis as the easy axis of the magnetization. This is in contradiction to the $b$-axis moment direction observed in the neutron diffraction data (Fig. 11). In order to account for this we have used a positive sign of $B_{2}{ }^{0}$, which does not change the calculated CEF excitations given in Fig. 15, but puts the moment in the $a b$ plane. The simulation shows a qualitative agreement with the experimental data giving an overall CEF splitting of $86.6 \mathrm{meV}$, which is in good agreement with the observed CEF splitting of $73.8 \mathrm{meV}$. We have calculated the single crystal (along all three directions) susceptibility and powder averaged susceptibility (see Fig. S7 of the SM [57]) as well as moments of the Dy ion using the CEF parameters based on the point charge model at $2 \mathrm{~K}$ in an applied field of $7 \mathrm{~T}$. The values of the Dy moment are $\mu_{x}=7.5 \mu_{\mathrm{B}}, \mu_{y}=6.6 \mu_{\mathrm{B}}, \mu_{z}=0.85 \mu_{\mathrm{B}}$, and the powder averaged moment $\mu_{\text {poly }}=4.98 \mu_{\mathrm{B}}$. The calculated powder average moment value of the Dy ion is slightly larger than the value of $4.25 \mu_{\mathrm{B}}$ (total moment from the $\mathrm{Dy}$ and $\mathrm{Ru}$ ) at $2 \mathrm{~K}$ and $7 \mathrm{~T}$ observed in the experimental data, which shows the limitation of the simple point charge model which does not include any covalence effects.

\section{CONCLUSIONS}

Our combined heat capacity, magnetization, $\mu$ SR, neutron diffraction, and inelastic neutron scattering results demonstrate that $\mathrm{Sr}_{2} \mathrm{DyRuO}_{6}$ (SDRO) exhibits a long-range ordered magnetic ground state below $40 \mathrm{~K}$. The heat capacity reveals a clear sign of two magnetic transitions, which are also indirectly supported through the magnetic susceptibility (both $\mathrm{AC}$ and DC) measurements. Our $\mu \mathrm{SR}$ and neutron diffraction studies provide direct support for long-range magnetic ordering below $40 \mathrm{~K}$. The neutron diffraction study shows that all the observed magnetic Bragg peaks between 2 and $40 \mathrm{~K}$ can be indexed using the magnetic propagation vector $k=(0,0,0)$. The magnetic structure shows that both the Dy and $\mathrm{Ru}$ atoms are arranged in type-I antiferromagnetic structure, which consists of interpenetrating sublattices of $\mathrm{Dy}^{3+}$ and $\mathrm{Ru}^{5+}$ atoms. In the $a b$ plane, the $\mathrm{Dy}^{3+}$ and $\mathrm{Ru}^{5+}$ moments are aligned AFM to each other, while along the $c$ axis they show FM coupling. Interestingly, the magnetic ordering is primarily governed by the $4 d$ moment on the $\mathrm{Ru}^{5+}$ atoms and the $\mathrm{Dy}^{3+}$ moments follow the Ru ordering at the same temperature $\left(T_{N 1}\right)$. In addition, it appears that the interactions responsible for the Dy ordering are weaker than the interactions responsible for the $\mathrm{Ru}$ ordering. The origin of the second anomaly in the heat capacity still remains an open question as the neutron diffraction study shows only one magnetic transition at $40 \mathrm{~K}$ and further single crystal neutron diffraction study will be important to understand the origin of the two phase transitions in SDRO. A possible cause for this second transition could reside in the appearance of an additional ferromagnetic component at $T_{N 2}$. From the inelastic neutron scattering study, we have estimated the spin gap of $3.25 \mathrm{meV}$ in the spin wave spectrum with a maximum zone boundary energy of $8.9 \mathrm{meV}$. Furthermore, we have also discussed the presence of crystal field excitations and their role in the observed reduced moment of the $\mathrm{Dy}^{3+}$ ions estimated through the neutron diffraction. The total CEF splitting observed in the experimental data agrees very well with that calculated using the point change model for the $\mathrm{Dy}^{3+}$ ion. The present work will generate interest in the condensed matter theory community to develop a realistic model to find out the common origin of the two magnetic phase transitions in the double perovskite family.

\section{ACKNOWLEDGMENTS}

We would like to thank L. Pascut for his help during the D20 experiment. S.S. wants to thank India-Nanomission, 
DST, and ISIS Facility for funding. D.T.A. and A.D.H. would like to thank CMPC-STFC, Grant No. CMPC- 515 09108, for financial support. We thank the ISIS facility for beam time for $\mu \mathrm{SR}$ and neutron measurements.
[1] L. Balents, Nature (London) 464, 199 (2010).

[2] S. T. Bramwell and M. J. P. Gingras, Science 294, 1495 (2001).

[3] P. Schiffer, A. P. Ramirez, D. A. Huse, P. L. Gammel, U. Yaron, D. J. Bishop, and A. J. Valentino, Phys. Rev. Lett. 74, 2379 (1995).

[4] Y. Zhou, K. Kanoda, and T.-K. Ng, Rev. Mod. Phys. 89, 025003 (2017).

[5] B. D. Gaulin, J. N. Reimers, T. E. Mason, J. E. Greedan, and Z. Tun, Phys. Rev. Lett. 69, 3244 (1992).

[6] C. M. Thompson, C. A. Marjerrison, A. Z. Sharma, C. R. Wiebe, D. D. Maharaj, G. Sala, R. Flacau, A. M. Hallas, Y. Cai, B. D. Gaulin, G. M. Luke, and J. E. Greedan, Phys. Rev. B 93, 014431 (2016).

[7] Y. Kojima, M. Watanabe, N. Kurita, H. Tanaka, A. Matsuo, K. Kindo, and M. Avdeev, Phys. Rev. B 98, 174406 (2018).

[8] O. Götze, D. J. J. Farnell, R. F. Bishop, P. H. Y. Li, and J. Richter, Phys. Rev. B 84, 224428 (2011).

[9] T. Aharen, J. E. Greedan, C. A. Bridges, A. A. Aczel, J. Rodriguez, G. MacDougall, G. M. Luke, T. Imai, V. K. Michaelis, S. Kroeker, H. Zhou, C. R. Wiebe, and L. M. D. Cranswick, Phys. Rev. B 81, 224409 (2010).

[10] S. Vasala and M. Karppien, Prog. Solid State Chem. 43, 1 (2014).

[11] A. A. Aczel, D. E. Bugaris, L. Li, J.-Q. Yan, C. de la Cruz, H.-C. zur Loye, and S. E. Nagler, Phys. Rev. B 87, 014435 (2013).

[12] P. D. Battle, T. C. Gibb, and C. W. Jones, J. Solid State Chem. 78, 281 (1989).

[13] E. Kermarrec, C. A. Marjerrison, C. M. Thompson, D. D. Maharaj, K. Levin, S. Kroeker, G. E. Granroth, R. Flacau, Z. Yamani, J. E. Greedan, and B. D. Gaulin, Phys. Rev. B 91, 075133 (2015).

[14] D. D. Russell, A. J. Neer, B. C. Melot, and S. Derakhshan, Inorg. Chem. 55, 2240 (2016).

[15] C. R. Wiebe, J. E. Greedan, G. M. Luke, and J. S. Gardner, Phys. Rev. B 65, 144413 (2002).

[16] M. P. Ghimire, L.-H. Wu, and X. Hu, Phys. Rev. B 93, 134421 (2016).

[17] O. N. Meetei, O. Erten, A. Mukherjee, M. Randeria, N. Trivedi, and P. Woodward, Phys. Rev. B 87, 165104 (2013).

[18] R. P. Borges, R. M. Thomas, C. Cullinan, J. M. D. Coey, R. Suryanarayanan, L. Ben-Dor, L. Pinsard-Gaudart, and A. Revcolevschi, J. Phys.: Condens. Matter 11, L445 (1999).

[19] Y. K. Wakabayashi, Y. Krockenberger, N. Tsujimoto, T. Boykin, S. Tsuneyuki, Y. Taniyasu, and H. Yamamoto, Nat. Commun. 10, 535 (2019).

[20] W. Westerburg, O. Lang, C. Ritter, C. Felser, W. Tremel, and G. Jakob, Solid State Commun. 122, 201 (2002).

[21] D. Serrate, J. M. De Teresa, P. A. Algarabel, J. Galibert, C. Ritter, J. Blasco, and M. R. Ibarra, Phys. Rev. B 75, 165109 (2007).

[22] J. Zhang, W.-J. Ji, J. Xu, X.-Y. Geng, J. Zhou, Z.-B. Gu, S.-H. Yao, and S.-T. Zhang, Sci. Adv. 3, e1701473 (2017).

[23] A. T. Lee and C. A. Marianetti, Phys. Rev. B 97, 045102 (2018).

[24] H. Chen, Quant. Mater. 3, 57 (2018).
[25] A. Kojima, K. Teshima, Y. Shirai, and T. Miyasaka, J. Am. Chem. Soc. 131, 6050 (2009).

[26] K.-I. Kobayashi, T. Kimura, H. Sawada, K. Terakura, and Y. Tokura, Nature (Lindon) 395, 677 (1998).

[27] H. Kato, T. Okuda, Y. Okimoto, and Y. Tomioka, Appl. Phys. Lett. 81, 328 (2002).

[28] D. Serrate, J. M. De Teresa, J. Blasco, M. R. Ibarra, L. Morellon, and C. Ritter, Eur. Phys. J. B 39, 35 (2004).

[29] J. M. De Teresa, D. Serrate, C. Ritter, J. Blasco, M. R. Ibarra, L. Morellon, and W. Tokarz, Phys. Rev. B 71, 092408 (2005).

[30] K. Samanta, P. Sanyal, and T. Saha-Dasgupta, Sci. Rep. 5, 15010 (2015).

[31] D. D. Sarma, P. Mahadevan, T. Saha-Dasgupta, S. Ray, and A. Kumar, Phys. Rev. Lett. 85, 2549 (2000).

[32] J. Kanamori and K. Terakura, J. Phys. Soc. Jpn. 70, 1433 (2001).

[33] D. Serrate, J. M. De Teresa, and M. R. Ibarra, J. Phys.: Condens. Matter 19, 023201 (2007).

[34] H. Kawanaka, I. Hase, S. Toyama, and Y. Nishihara, J. Phys. Soc. Jpn. 68, 2890 (1999).

[35] P. L. Bernardo, L Ghivelder, H. S. Amorim, J. J. Neumeier, and S García, New J. Phys. 17, 103007 (2015).

[36] E. Granado, J. W. Lynn, R. F. Jardim, and M. S. Torikachvili, Phys. Rev. Lett. 110, 017202 (2013).

[37] R. P. Singh and C. V. Tomy, J. Phys.: Condens. Matter 20, 235209 (2008).

[38] N. G. Parkinson, P. D. Hatton, J. A. K. Howard, C. Ritter, R. M. Ibberson, and M.-K. Wu, J. Phys.: Condens. Matter 16, 7611 (2004).

[39] P. D. Battle and C. W. Jones, J. Solid State Chem. 78, 108 (1989).

[40] A. A. Aczel, P. J. Baker, D. E. Bugaris, J. Yeon, H.-C. zur Loye, T. Guidi, and D. T. Adroja, Phys. Rev. Lett. 112, 117603 (2014).

[41] P. Mohanty, S. Marik, D. Singh, and R. P. Singh, Mater. Res. Express 4, 126103 (2017).

[42] C. A. Triana, D. A. L. Téllez, J. A. Rodríguez, F. Fajardo, and J. Roa-Rojas, Materials Lett. 82, 116 (2012).

[43] C. A. Triana, D. A. Landínez Téllez, and J. Roa-Rojas, Mater. Charact. 99, 128 (2015).

[44] J. Rodriguez-Carvajal, Physica B 192, 55 (1993).

[45] R. P. Singh and C. V. Tomy, Solid State Commun. 150, 804 (2010).

[46] Y. Doi, Y. Hinatsu, K.-I. Oikawa, Y. Shimojob, and Y. Morii, J. Mater. Chem. 10, 797 (2000).

[47] R. Kumar, C. V. Tomy, R. Nagarajan, P. L. Paulose, and S. K. Malik, Physica B 404, 2369 (2009).

[48] A. E. Taylor, R. Morrow, M. D. Lumsden, S. Calder, M. H. Upton, A. I. Kolesnikov, M. B. Stone, R. S. Fishman, A. Paramekanti, P. M. Woodward, and A. D. Christianson, Phys. Rev. B 98, 214422 (2018).

[49] A. S. Cavichini, M. T. Orlando, J. B. Depianti, J. L. Passamai, Jr., F. Damay, F. Porcher, and E. Granado, Phys. Rev. B 97, 054431 (2018). 
[50] B. D. Cullity and C. D. Graham, Introduction to Magnetic Materials, 2nd ed. (John Wiley and Sons, Hoboken, NJ, 2009).

[51] A. Bhattacharyya, D. T. Adroja, M. Smidman, and V. K. Anand, Sci. China: Phys. Mech. Astron. 61, 127402 (2018).

[52] P. Yadav, S. Sharma, P. J. Baker, P. K. Biswas, I. da Silva, R. Raghunathan, U. Deshpande, R. J. Choudhary, N. P. Lalla, and A. Banerjee, Phys. Rev. B 99, 214421 (2019).

[53] A. A. Aczel, Z. Zhao, S. Calder, D. T. Adroja, P. J. Baker, and J.-Q. Yan, Phys. Rev. B 93, 214407 (2016).

[54] C. R. Wiebe, J. E. Greedan, P. P. Kyriakou, G. M. Luke, J. S. Gardner, A. Fukaya, I. M. Gat-Malureanu, P. L. Russo, A. T. Savici, and Y. J. Uemura, Phys. Rev. B 68, 134410 (2003).

[55] J. Sannigrahi, D. T. Adroja, C. Ritter, W. Kockelmann, A. D. Hillier, K. S. Knight, A. T. Boothroyd, M. Wakeshima, Y. Hinatsu, F. Mosselmans, and S. Ramos, Phys. Rev. B 99, 184440 (2019).

[56] S. J. Blundell, Contemp. Phys. 40, 175 (1999).

[57] See Supplemental Material at http://link.aps.org/supplemental/ 10.1103/PhysRevB.101.094413 for muon spin rotation and neutron scattering investigations of the $B$-site ordered double perovskite $\mathrm{Sr}_{2} \mathrm{DyRuO}_{6}$.

[58] J. Rodriguez-Carvajal, BASIREPS: A program for calculating irreducible representations of space groups and basis functions for axial and polar vector properties. Part of the FullProf Suite of programs available at http://www.ill.eu/sites/fullprof/.

[59] C. Ritter, Solid State Phenom. 170, 263 (2011).

[60] N. G. Parkinson, P. D. Hatton, J. A. K. Howard, C. Ritter, F. Z. Chien, and M.-K. Wu, J. Mater. Chem. 13, 1468 (2003).

[61] R. Sáez-Puche, E. Climent-Pascual, E. Climent-Pascual, R. Ruiz-Bustos, and M. T. Fernández-Díaz, Prog. Solid State Chem. 35, 211 (2007).
[62] G. Cao, S. McCall, Z. X. Zhou, C. S. Alexander, and J. E. Crow, Phys. Rev. B 63, 144427 (2001).

[63] G. Cao, S. McCall, Z. X. Zhou, C. S. Alexander, J. E. Crow, and R. P. Guertin, J. Magn. Magn. Mate. 226-230, 218 (2001).

[64] R. P. Guertin and S. McCall, Phys. Rev. B 65, 184413 (2002).

[65] V. K. Anand, A. D. Hillier, D. T. Adroja, D. D. Khalyavin, P. Manuel, G. Andre, S. Rols, and M. M. Koza, Phys. Rev. B 97, 184422 (2018)

[66] S. A. Ivanov, C. Ritter, P. Nordblad, R. Tellgren, M. Weil, V. Carolus, Th. Lottermoser, M. Fiebig, and R. Mathieu, J. Phys. D: Appl. Phys. 50, 085001 (2017).

[67] H. S. Jeevan, D. T. Adroja, A. D. Hillier, Z. Hossain, C. Ritter, and C. Geibel, Phys. Rev. B 84, 184405 (2011).

[68] D. T. Adroja et al. (unpublished); HET experimental report, ISIS Facility (2008); R. Singh, Ph.D. thesis, IIT, Bombay, 2010.

[69] D. D. Maharaj, G. Sala, C. A. Marjerrison, M. B. Stone, J. E. Greedan, and B. D. Gaulin, Phys. Rev. B 98, 104434 (2018).

[70] A. A. Aczel, A. M. Cook, T. J. Williams, S. Calder, A. D. Christianson, G.-X. Cao, D. Mandrus, Y.-B. Kim, and A. Paramekanti, Phys. Rev. B 93, 214426 (2016).

[71] P. Carlo, J. P. Clancy, K. Fritsch, C. A. Marjerrison, G. E. Granroth, J. E. Greedan, H. A. Dabkowska, and B. D. Gaulin, Phys. Rev. B 88, 024418 (2013).

[72] S. M. Disseler, J. W. Lynn, R. F. Jardim, M. S. Torikachvili, and E. Granado, Phys. Rev. B 93, 140407(R) (2016).

[73] A. E. Taylor, R. Morrow, R. S. Fishman, S. Calder, A. I. Kolesnikov, M. D. Lumsden, P. M. Woodward, and A. D. Christianson, Phys. Rev. B 93, 220408(R) (2016).

[74] N. Kumar and A. Sundaresan, Solid Sate Commun. 150, 1162 (2010).

[75] O. Arnold et al., Nucl. Instrum. Methods Phys. Res. Sect. A 764, 156 (2014). 